\title{
Degenerate limit thermodynamics beyond leading order for models of dense matter
}

\author{
Constantinos Constantinou ${ }^{1}$ \\ Institute for Advanced Simulation, Institut für Kernphysik, and Jülich Center \\ for Hadron Physics, Forschungszentrum Jülich, D-52425 Jülich, Germany \\ Brian Muccioli $^{2}$ \\ Department of Physics and Astronomy, Ohio University, Athens, OH 45701 \\ Madappa Prakash ${ }^{3}$ \\ Department of Physics and Astronomy, Ohio University, Athens, OH 45701 \\ James M. Lattimer ${ }^{4}$ \\ Department of Physics and Astronomy, Stony Brook University, Stony Brook, NY \\ 11794-3800
}

\begin{abstract}
Analytical formulas for next-to-leading order temperature corrections to the thermal state variables of interacting nucleons in bulk matter are derived in the degenerate limit. The formalism developed is applicable to a wide class of nonrelativistic and relativistic models of hot and dense matter currently used in nuclear physics and astrophysics (supernovae, proto-neutron stars and neutron star mergers) as well as in condensed matter physics. We consider the general case of arbitrary dimensionality of momentum space and an arbitrary degree of relativity (for relativistic models). For non-relativistic zero-range interactions, knowledge of the Landau effective mass suffices to compute next-to-leading order effects, but for finite-range interactions, momentum derivatives of the Landau effective mass function up to second order are required. Results from our analytical formulas are compared with the exact results for zero- and finite-range potential and relativistic mean-field theoretical models. In all cases, inclusion of next-to-leading order temperature effects substantially extends the ranges of partial degeneracy for which the analytical treatment remains valid. Effects of many-body correlations that deserve further investigation are highlighted.
\end{abstract}

\footnotetext{
${ }^{1}$ c.constantinou@fz-juelich.de

2bm956810@ohio.edu

3 prakash@ohio.edu

4 james.lattimer@stonybrook.edu
} 
Keywords: Hot and dense matter, thermal effects, potential and field-theoretical models.

\section{INTRODUCTION}

Homogeneous bulk matter comprised of fermions is commonly encountered in astrophysics, condensed matter physics, and nuclear physics. For extreme degenerate to near-degenerate conditions which prevail when the temperature is

5 small compared to the Fermi temperature, Landau's Fermi Liquid Theory (FLT) has been a useful guide to describe the thermodynamic and transport properties of the system (see, e.g., [1] and references therein). The equation of state (EOS) of dense matter in cold and catalyzed neutron stars, for example, is dominated by the zero-temperature properties (which predominantly determine the

10 structure of and neutrino interactions within the star) while finite-temperature corrections (important for the cooling of neutron stars) are adequately given by the degenerate limit expressions from FLT. The leading order FLT corrections to the energy density and pressure are quadratic in the temperature; corrections to the entropy and specific heats are linear in the temperature. However, matter 15 in supernovae and proto-neutron stars [2, 3], especially in situations in which collapse to a black hole occurs, may reach temperatures exceeding the Fermi temperature, in which case the finite-temperature contributions extend beyond those given by the FLT. In neutron star mergers, it is likely that in some cases a hyper-massive neutron star, or HMNS, is formed: the merged remnant mass ex20 ceeds the cold maximum mass. The metastable support is provided by rotation, including differential rotation, and thermal effects. The timescale over which collapse to a black hole eventually occurs, potentially observable in gravitational wave signatures, will therefore be sensitive to thermal effects [4. In this contribution, we derive analytical formulas for next-to-leading order temperature 25 effects in the state variables of interacting nucleons in both the non-relativistic and relativistic limits for a variety of nuclear interaction models.

For non-relativistic models with zero-range interactions, knowledge of the Landau effective mass is sufficient to satisfy thermodynamic identities. However, in the general case of finite-range interactions, momentum derivatives of

so the Landau effective mass function up to second order are required. We compare results from the analytical expressions to exact numerical calculations for zeroand finite-range potential models as well as for relativistic mean-field theoretical models. The analytic next-to-leading order expressions lead to an improvement of the leading order results of FLT, as demonstrated by the wider ranges of de35 generacy and temperature for which they remain valid. In addition, we derive relations in a form that are independent of the dimensionality of the momentum space under consideration. Therefore, although our discussion focuses on examples from dense matter physics, which are three-dimensional systems in momentum space, the expressions derived can have a wider application to cer-

40 tain problems in condensed matter physics in which the momentum space is two-dimensional. 
The paper is organized as follows. In Sec. 2, the formalism to calculate next-to-leading order corrections to the results of FLT in D-dimensions is developed. Effects that lie beyond the formalism employed here and deserve further investigation are highlighted at the end of this section. Analytical formulas appropriate for 3-dimensions including exchange contributions (Hartree-Fock) are given in Sec. 3, whereas Sec. 4 contains results for 2-dimensions. The formalism is applied to zero- and finite-range potential models and relativistic field-theoretical models in Sec. 5. Section 6 summarizes contributions from lep-

${ }_{50}$ tons and photons required in astrophysical applications. Numerical results for illustrative models are presented in Sec. 7 where the extent to which the nextto-leading order corrections improve the FLT results are demonstrated. Section 8 presents a summary and conclusions. Useful formulas for the evaluation of the thermal properties are provided in Appendices A, B, C, and D.

\section{GENERAL CONSIDERATIONS}

For a generic Hamiltonian density $\mathcal{H}(n, \tau)$ where $n$ and $\tau$ are the number and kinetic energy densities respectively, the single-particle potential $U$ is obtained from a functional differentiation of $\mathcal{H}$ with respect to $n$, and can contain terms depending on $n$ as well as the momentum $p$ :

$$
U(n, p)=\frac{\delta \mathcal{H}}{\delta n}=\mathcal{U}(n)+R(p),
$$

60

where $\mathcal{U}(n)$ denotes contributions that depend on $n$ only. Note that $R$ above may also be $n$-dependent but we will suppress this for notational simplicity.

The study of the thermodynamic properties of a fermion system involves integrals of the form

$$
I=\int_{0}^{\infty} d p g(p) f(p), \quad f(p)=\left[1+\exp \left(\frac{\epsilon-\mu}{T}\right)\right]^{-1},
$$

where $T$ is the temperature, $\mu$ is the chemical potential, and the single-particle 65 spectrum

$$
\epsilon=\frac{p^{2}}{2 m}+U(n, p)
$$

The structure of the function $g(p)$ is determined by the state variable under consideration. In general, integrals involving the Fermi function $f(p)$ do not admit analytical solutions and thus require numerical treatment. In the lowtemperature limit, however, when the degeneracy parameter

$$
\eta=\frac{\mu-\epsilon(p=0)}{T}
$$

70 is large, these integrals can be approximately evaluated employing the Sommerfeld expansion (see, e.g., [5]) by transforming Eq. (2) to

$$
I=\int_{0}^{\infty} d y \frac{\phi(y)}{1+\exp (y-\eta)} \stackrel{\eta \gg 1}{\longrightarrow} \int_{0}^{\eta} \phi(y) d y+\left.\frac{\pi^{2}}{6} \frac{d \phi}{d y}\right|_{y=\eta}+\left.\frac{7 \pi^{4}}{360} \frac{d^{3} \phi}{d y^{3}}\right|_{y=\eta}+\ldots
$$


with the identification $\phi(y) d y=g(p) d p$, and the substitution

$$
y \equiv \frac{\epsilon-\mathcal{U}(n)}{T}=\frac{p^{2}}{2 m T}+\frac{R(p)}{T},
$$

from which it follows that

$$
\frac{d y}{d p}=\frac{p}{\mathcal{M} T} \quad \text { and } \quad \phi(y)=\frac{\mathcal{M} T}{p} g(p),
$$

where the Landau effective mass function

$$
\mathcal{M}(p)=m\left[1+\frac{m}{p} \frac{d R(p)}{d p}\right]^{-1} .
$$

75 This function is implicitly temperature-dependent and its relation to the Landau effective mass $m^{*}$ is

$$
\mathcal{M}\left(p=p_{F} ; T=0\right)=m^{*},
$$

where $p_{F}$ is the Fermi momentum. From the relations in Eq. (7)

$$
\begin{aligned}
\frac{d \phi}{d y} & =-\frac{T^{2} \mathcal{M}^{2} g}{p^{3}}\left[1-p\left(\frac{g^{\prime}}{g}+\frac{\mathcal{M}^{\prime}}{\mathcal{M}}\right)\right] \\
\frac{d^{3} \phi}{d y^{3}} & =-\frac{15 T^{4} \mathcal{M}^{4} g}{p^{7}}\left[1-p\left(\frac{g^{\prime}}{g}+\frac{5}{3} \frac{\mathcal{M}^{\prime}}{\mathcal{M}}\right)\right. \\
& +\frac{2}{5} p^{2}\left(\frac{g^{\prime \prime}}{g}+\frac{11}{3} \frac{\mathcal{M}^{\prime} g^{\prime}}{\mathcal{M} g}+\frac{11}{6} \frac{\mathcal{M}^{\prime 2}}{\mathcal{M}^{2}}+\frac{7}{6} \frac{\mathcal{M}^{\prime \prime}}{\mathcal{M}}\right) \\
& -\frac{p^{3}}{15}\left(\frac{g^{\prime \prime \prime}}{g}+7 \frac{\mathcal{M}^{\prime 2} g^{\prime}}{\mathcal{M}^{2} g}+6 \frac{\mathcal{M}^{\prime} g^{\prime \prime}}{\mathcal{M} g}+4 \frac{\mathcal{M}^{\prime \prime} g}{\mathcal{M} g}\right. \\
+ & \left.\left.\frac{\mathcal{M}^{\prime 3}}{\mathcal{M}^{3}}+\frac{\mathcal{M}^{\prime \prime \prime}}{\mathcal{M}}+4 \frac{\mathcal{M}^{\prime} \mathcal{M}^{\prime \prime}}{\mathcal{M}^{2}}\right)\right],
\end{aligned}
$$

where the primes denote differentiation with respect to $p$.

For a system in $D$ dimensions having $\gamma$ internal degrees of freedom, the number density is given by

$$
n=C_{D} \int d p p^{D-1} f_{p} \quad \text { with } \quad C_{D}=\frac{\gamma}{(2 \pi \hbar)^{D}} \frac{D \pi^{D / 2}}{(D / 2) !} .
$$

The combination of Eqs. (5), 10, and (11) with $g_{n}=p^{D-1}$ results in

$$
\begin{aligned}
n & =\frac{C_{D}}{D}\left\{p_{\mu}^{D}+\frac{\pi^{2}}{6} D p_{\mu}^{D-4} \mathcal{M}_{\mu}^{2} T^{2}\left(D-2+p_{\mu} \frac{\mathcal{M}_{\mu}^{\prime}}{\mathcal{M}_{\mu}}\right)\right. \\
& +\frac{7 \pi^{4}}{360} D p_{\mu}^{D-8} \mathcal{M}_{\mu}^{4} T^{4}[(D-6)(D-4)(D-2) \\
& +\left(\frac{p_{\mu} \mathcal{M}_{\mu}^{\prime}}{\mathcal{M}_{\mu}}\right)^{3}+(7 D-18)\left(\frac{p_{\mu} \mathcal{M}_{\mu}^{\prime}}{\mathcal{M}_{\mu}}\right)^{2}
\end{aligned}
$$




$$
\begin{aligned}
& +\quad\left(6 D^{2}-40 D+59\right) \frac{p_{\mu} \mathcal{M}_{\mu}^{\prime}}{\mathcal{M}_{\mu}}+\frac{p_{\mu}^{3} \mathcal{M}_{\mu}^{\prime \prime \prime}}{\mathcal{M}_{\mu}} \\
& \left.\left.+\quad 4 \frac{p_{\mu}^{3} \mathcal{M}_{\mu}^{\prime} \mathcal{M}_{\mu}^{\prime \prime}}{\mathcal{M}_{\mu}^{2}}+(4 D-11) \frac{p_{\mu}^{2} \mathcal{M}_{\mu}^{\prime \prime}}{\mathcal{M}_{\mu}}\right]+\ldots\right\},
\end{aligned}
$$

where the subscript $\mu$ denotes quantities evaluated at $\epsilon=\mu$, i.e.,

$$
\epsilon=\mu=\frac{p_{\mu}^{2}}{2 m}+R\left(p_{\mu}\right)+\mathcal{U}(n) .
$$

For $N$ particles in a volume $V$, the number density $n=N / V$ at $T=0$ and at ${ }_{85}$ finite $T$ is the same. Equating the result in Eq. 13 to its $T=0$ counterpart $n=C_{D} p_{F}^{3} / D$, and perturbatively inverting we get

$$
p_{\mu}=p_{F}\left[1-\frac{\pi^{2}}{6} \frac{m^{* 2} T^{2}}{p_{F}^{4}}\left(D-2+\frac{p_{F} \mathcal{M}_{F}^{\prime}}{m^{*}}\right)+\ldots\right]
$$

with

$$
p_{F}=\left(\frac{n D}{C_{D}}\right)^{1 / D} \quad \text { and } \quad \mathcal{M}_{F}^{\prime}=\left.\frac{d \mathcal{M}}{d p}\right|_{p_{F}} .
$$

As our main goal here is to derive the next-to-leading order correction in temperature for the entropy density $s$, it suffices to truncate the series expansion of

90 $p_{\mu}$ to $\mathcal{O}\left(T^{2}\right)$. We will show below that higher-order terms do not contribute at this level of approximation where we may also neglect the temperature dependence of $\mathcal{M}$ and its derivatives. The result in Eq. (15) helps us to work only with quantities defined on the Fermi surface as done in Landau's Fermi-Liquid theory [1, 6, 17. The entropy density is formally given by

$$
s=-C_{D} \int d p p^{D-1}\left[f_{p} \ln f_{p}-\left(1-f_{p}\right) \ln \left(1-f_{p}\right)\right] .
$$

Integrating this expression twice by parts we obtain

$$
s=\frac{1}{T}\left\{\frac{\tau}{m}\left(\frac{1}{2}+\frac{1}{D}\right)+n(\mathcal{U}-\mu)+C_{D} \int d p p^{D-1} f_{p}\left[R(p)+\frac{p}{D} \frac{d R(p)}{d p}\right]\right\}
$$

where

$$
\tau=C_{D} \int d p p^{D+1} f_{p}
$$

is the kinetic energy density. With the aid of Eq. (14) for the chemical potential, Eq. 18 can be written as

$$
s=\frac{1}{T}\left\{\frac{\tau}{m}\left(\frac{1}{2}+\frac{1}{D}\right)-n \frac{p_{\mu}^{2}}{2 m}+C_{D} \int d p p^{D-1} f_{p}\left[R(p)-R\left(p_{\mu}\right)+\frac{p}{D} \frac{d R(p)}{d p}\right]\right\}
$$


from which we identify the functions

$$
\begin{aligned}
g_{1 s}(p) & =\frac{p^{D+1}}{m}\left(\frac{1}{2}+\frac{1}{D}\right)-p^{D-1} \frac{p_{\mu}^{2}}{2 m} \\
g_{2 s}(p) & =p^{D-1}\left[R(p)-R\left(p_{\mu}\right)+\frac{p}{D} \frac{d R(p)}{d p}\right]
\end{aligned}
$$

100

to be used in the Sommerfeld expansion. For both of these functions, the first term on the right-hand side of Eq. (5) involving an integral vanishes yielding

$$
\begin{aligned}
s & =\frac{\pi^{2}}{3} C_{D} p_{\mu}^{D-2} \mathcal{M}_{\mu} T+\frac{7 \pi^{4}}{90} C_{D} p_{\mu}^{D-6} \mathcal{M}_{\mu}^{3} T^{3}[(D-4)(D-2) \\
& \left.+p_{\mu}^{2} \frac{\mathcal{M}_{\mu}^{\prime 2}}{\mathcal{M}_{\mu}^{2}}+p_{\mu}^{2} \frac{\mathcal{M}_{\mu}^{\prime \prime}}{\mathcal{M}_{\mu}}+(3 D-7) p_{\mu} \frac{\mathcal{M}_{\mu}^{\prime}}{\mathcal{M}_{\mu}}\right] .
\end{aligned}
$$

Use of Eqs. (15) and 16 in the above result delivers the working expression for $s$ in terms of quantities defined on the Fermi surface:

$$
\begin{aligned}
s & \simeq \frac{\pi^{2}}{3} C_{D} p_{F}^{D-2} m^{*} T+\frac{\pi^{4}}{45} C_{D} p_{F}^{D-6} m^{* 3} T^{3}[(D-9)(D-2) \\
& \left.+\frac{7}{2} p_{F}^{2} \frac{\mathcal{M}_{F}^{\prime 2}}{m^{* 2}}+\frac{7}{2} p_{F}^{2} \frac{\mathcal{M}_{F}^{\prime \prime}}{m^{*}}+\frac{(16 D-39)}{2} p_{F} \frac{\mathcal{M}_{F}^{\prime}}{m^{*}}\right]
\end{aligned}
$$

where the $\mathcal{O}(T)$ term is the well known result from FLT. We note that a large number of cancellations occur in obtaining Eqs. (23) and (24) despite the complexity of of Eqs. (10) and (11). For a system composed of different kinds of particles the total entropy density is a sum of the contributions from the individual species where, in Eq. 24, the Fermi momentum, the effective mass, and its derivatives all carry a particle-species index $i$.

110 Equation 24 forms the basis from which other properties of the system can be derived. For example, the entropy per particle is the simple ratio $S=s / n$, whereas the thermal energy, pressure, and chemical potential can be obtained through the application of the appropriate Maxwell relations [5]:

$$
E_{t h}=\int T d S, \quad P_{t h}=-n^{2} \int \frac{d S}{d n} d T \quad \text { and } \quad \mu_{t h}=-\int \frac{d s}{d n} d T,
$$

where the integrals above are performed at constant density [for a multiple115 species system, $\left.\mu_{t h, i}=-\int\left(d s / d n_{i}\right) d T\right]$.

The specific heats at constant volume and pressure are given by the standard thermodynamics expressions [5]

$$
\begin{aligned}
C_{V} & =\left.T \frac{\partial S}{\partial T}\right|_{n}=\left.\frac{\partial E_{t h}}{\partial T}\right|_{n} \\
C_{P} & =\left.T \frac{\partial S}{\partial T}\right|_{P}=C_{V}+\frac{T}{n^{2}} \frac{\left(\left.\frac{\partial P_{t h}}{\partial T}\right|_{n}\right)^{2}}{\left.\frac{\partial P}{\partial n}\right|_{T}}
\end{aligned}
$$


We note that the formalism above has not considered effects, for example, from long-wavelength fluctuations, from single particle-hole excitations, or from collective and pairing correlations near the Fermi surface [1, 8, 9, 10]. Effects of correlations generally lead to an enhancement of the effective mass at the Fermi surface (see also Ref. [11]) and also influence the entropy. These correlations are not captured in mean-field theories even when exchange contributions (in both non-relativistic and relativistic models) are included. Pethick and Carneiro [8]

125 have shown that long-wavelength fluctuations lead to non-analytic behavior of the quasiparticle interaction which in turn gives rise to $T^{3} \ln T$ terms in the specific heat and entropy. Such terms then become the leading correction to the results of Fermi-liquid theory at low $T$. In liquid ${ }^{3} \mathrm{He}$, use of only $T$ and $T^{3} \ln T$ terms gives a remarkably good fit to specific heat data (see Fig. 1.8 in Ref. 1). In 2-dimensional Fermi systems with contact interactions, non-analytic corrections to the real part of the self-energy lead to $T^{2}$ contributions which overwhelm the $T^{3}$ terms to the entropy at low $T[10$.

In dense nuclear matter, however, the enhancement of the effective mass appears to be much smaller than in liquid ${ }^{3} \mathrm{He}$ as found by Fantoni et al. [12,

135 who used correlated basis functions (CBF) to study the nucleon optical potential in nuclear matter. A comparison of single-particle energies for variational and variational plus second-order CBF calculations made in Figure 6 of Ref. [12] does not show significant differences. Since the enhancement of the effective mass and the $T^{3} \ln T$ term have the same origin, it is likely that contributions from the $T^{3} \ln T$ term are not large in dense nuclear matter. However, this topic deserves further scrutiny in light of modern developments in nuclear theory. Contributions from these sources must be added to those considered in this work when deemed appropriate.

\section{RESULTS FOR $D=3$}

145 For a single-species system of spin $1 / 2$ particles in 3 dimensions [for which $\left.C_{3}=1 /\left(\pi^{2} \hbar^{3}\right)\right]$, the entropy density becomes

$$
s=\frac{p_{F} m^{*} T}{3 \hbar^{3}}-\frac{2 \pi^{2}}{15 \hbar^{3}} \frac{m^{* 3} T^{3}}{p_{F}^{3}}\left(1-L_{F}\right),
$$

where

$$
L_{F} \equiv \frac{7}{12} p_{F}^{2} \frac{\mathcal{M}_{F}^{\prime 2}}{m^{* 2}}+\frac{7}{12} p_{F}^{2} \frac{\mathcal{M}_{F}^{\prime \prime}}{m^{*}}+\frac{3}{4} p_{F} \frac{\mathcal{M}_{F}^{\prime}}{m^{*}} .
$$

We stress that, in general,

$$
\left.\frac{d \mathcal{M}(p)}{d p}\right|_{p_{F}}=\mathcal{M}_{F}^{\prime} \neq m^{* \prime}=\frac{d \mathcal{M}\left(p_{F}\right)}{d p_{F}}
$$

as $R$ can contain both $p$ and $p_{F}$ (via $n$ ). In terms of the level-density parameter ${ }_{150} a=\pi^{2} m^{*} /\left(2 p_{F}^{2}\right)=\pi^{2} /\left(4 T_{F}\right)$ (where $T_{F}$ is the Fermi temperature), Eq. 28 can be written as

$$
s=2 a n T-\frac{16}{5 \pi^{2}} a^{3} n T^{3}\left(1-L_{F}\right) .
$$


The quantity $L_{F}$ arises from nontrivial momentum dependencies in the singleparticle potential. For free gases (where $R(p)=0$ ), and for systems having only contact interactions where $R(p) \propto p^{2}$ (such as Skyrme models), $L_{F}=0$.

Equation (31) in conjunction with Eqs. (25)-(27) leads to

$$
\begin{aligned}
S & =2 a T-\frac{16}{5 \pi^{2}} a^{3} T^{3}\left(1-L_{F}\right), \quad E_{t h}=a T^{2}-\frac{12}{5 \pi^{2}} a^{3} T^{4}\left(1-L_{F}\right) \\
E_{t h} & =a T^{2}-\frac{12}{5 \pi^{2}} a^{3} T^{4}\left(1-L_{F}\right) \\
P_{t h} & =\frac{2}{3} a n Q T^{2}-\frac{8}{5 \pi^{2}} a^{3} n Q T^{4}\left(1-L_{F}+\frac{n}{2 Q} \frac{d L_{F}}{d n}\right) \\
\mu_{t h} & =-a\left(1-\frac{2 Q}{3}\right) T^{2}+\frac{4}{5 \pi^{2}} a^{3} T^{4}\left[\left(1-L_{F}\right)(1-2 Q)-n \frac{d L_{F}}{d n}\right] \\
C_{V} & =2 a T-\frac{48}{5 \pi^{2}} a^{3} T^{3}\left(1-L_{F}\right) \quad \text { and } \quad C_{P}=C_{V}+\frac{16}{9} \frac{a^{2} Q^{2} T^{3}}{\frac{d P_{0}}{d n}}
\end{aligned}
$$

where

$$
Q=1-\frac{3 n}{2 m^{*}} \frac{d m^{*}}{d n} .
$$

In the derivation of Eq. (36) we have assumed that the zero-temperature pressure $P_{0}$ is such that $d P_{0} / d n \gg d P_{t h} / d n$. This condition will not be met in situations where $P_{0}$ is relatively flat as in the vicinity of a critical point. When this is the case, we must use Eq. (27) for $C_{P}$, with

$$
\begin{aligned}
\left(\left.\frac{\partial P_{t h}}{\partial T}\right|_{n}\right)^{2} & =\left(\frac{4}{3} a n Q T\right)^{2}\left[1-\frac{48}{5 \pi^{2}} a^{2} T^{2}\left(1-L_{F}+\frac{n}{2 Q} \frac{d L_{F}}{d n}\right)\right] \\
\left.\frac{\partial P}{\partial n}\right|_{T} & =\frac{d P_{0}}{d n}+\frac{2}{3} a Q T^{2}\left(1-\frac{2 Q}{3}+n \frac{d Q}{d n}\right) \\
& -\frac{5}{8 \pi^{2}} a^{3} Q T^{4}\left[\left(1-2 Q+n \frac{d Q}{d n}\right)\left(1-L_{F}+\frac{n}{2 Q} \frac{d L_{F}}{d n}\right)\right. \\
& \left.-n \frac{d L_{F}}{d n}\left(1-2 Q+\frac{n}{2 Q^{2}} \frac{d Q}{d n}\right)+\frac{n^{2}}{2 Q} \frac{d^{2} L_{F}}{d n^{2}}\right] .
\end{aligned}
$$

Similar considerations as with Eq. (36) hold for the ratio of the specific heats

$$
\frac{C_{P}}{C_{V}}=1+\frac{8}{9} \frac{a Q^{2} T^{2}}{\frac{d P_{0}}{d n}} .
$$

Other quantities of interest in astrophysical applications include the thermal index $\Gamma_{t h}$

$$
\Gamma_{t h}=1+\frac{P_{t h}}{n E_{t h}}=1+\frac{2 Q}{3}-\frac{4}{5 \pi^{2}} a^{2} n T^{2} \frac{d L_{F}}{d n}
$$

and the adiabatic index $\Gamma_{S}$

$$
\Gamma_{S}=\left.\frac{C_{P}}{C_{V}} \frac{n}{P} \frac{\partial P}{\partial n}\right|_{T}=\frac{n}{P_{0}}\left[\frac{d P_{0}}{d n}+\frac{2}{3} a Q T^{2}\left(1+\frac{2 Q}{3}+n \frac{d Q}{d n}-\frac{n}{P_{0}} \frac{d P_{0}}{d n}\right)\right]
$$


where, in addition to Eqs. (34), 39 and $(40)$, the approximation

$$
\frac{1}{P} \simeq \frac{1}{P_{0}}\left(1-\frac{P_{t h}}{P_{0}}\right)
$$

was used. In its native variables $(n, S), \Gamma_{S}$ is given by

$$
\Gamma_{S}=\left.\frac{n}{P} \frac{\partial P}{\partial n}\right|_{S}=\frac{n}{P_{0}+\frac{n Q S^{2}}{6 a}}\left[\frac{d P_{0}}{d n}+\frac{Q S^{2}}{6 a}\left(1+\frac{2}{3} Q+\frac{n}{Q} \frac{d Q}{d n}\right)\right] .
$$

To arrive to Eq. (44) one begins by inverting Eq. 32 for the small parameter

$$
a T=\frac{S}{2}+\frac{S^{3}}{5 \pi^{2}}\left(1-L_{F}\right)
$$

which is then employed in the expression for the thermal pressure with the results

$$
\begin{aligned}
P_{t h} & =\frac{n Q}{6 a} S^{2}+\frac{n Q}{30 \pi^{2} a} S^{4}\left(1-L_{F}-\frac{3 n}{2 Q} \frac{d L_{F}}{d n}\right) \\
\left.\frac{\partial P_{t h}}{\partial n}\right|_{S} & =\frac{Q}{6 a} S^{2}\left(1+\frac{2}{3} Q+\frac{n}{Q} \frac{d Q}{d n}\right) \\
& +\frac{Q}{30 \pi^{2} a} S^{4}\left[\left(1+\frac{2}{3} Q+\frac{n}{Q} \frac{d Q}{d n}\right)\left(1-L_{F}\right)\right. \\
& \left.-2 n \frac{d L_{F}}{d n}\left(1+\frac{3}{2 Q}\right)-\frac{3 n^{2}}{2 Q} \frac{d^{2} L_{F}}{d n^{2}}\right]
\end{aligned}
$$

Finally, the result is truncated to $\mathcal{O}\left(S^{2}\right)$ in both the numerator as well as the denominator. We refrain from invoking approximation (43) as for nuclear systems, $P_{0}$ can cross 0 at low densities. This is not a problem in the variables $(n, T)$ because the degenerate approximation breaks down at sufficiently low density regardless of $T$. In the variables $(n, S)$, however, for small values of the entropy

175 the system remains degenerate irrespective of the density, and thus division by zero is avoided (as could happen if Eq. (43) is used).

We point out that the adiabatic index is related to the squared speed of sound $c_{s}$ according to

$$
\left(\frac{c_{s}}{c}\right)^{2}=\Gamma_{S} \frac{P}{h+m n}
$$

where $h=n E+P$ is the enthalpy density.

\section{RESULTS FOR $D=2$}

In condensed matter physics, 2-dimensional systems are of much interest. In the current framework, the entropy density is

$$
s=\frac{\pi^{2}}{3} C_{2} m^{*} T+\frac{7 \pi^{4}}{90} C_{2} \frac{m^{* 3} T^{3}}{p_{F}^{4}}\left(p_{F}^{2} \frac{\mathcal{M}_{F}^{\prime 2}}{m^{* 2}}+p_{F}^{2} \frac{\mathcal{M}_{F}^{\prime \prime}}{m^{*}}-p_{F} \frac{\mathcal{M}_{F}^{\prime}}{m^{*}}\right)
$$


with $C_{2}=\left(1 / 2 \pi \hbar^{2}\right)$. A noteworthy feature of this result is that the $\mathcal{O}\left(T^{3}\right)$ term receives contributions only from the derivatives of the effective mass function with respect to $p$ at the Fermi surface. Thus, it is absent not only for free gases but also for systems with contact interactions where the $p^{2}$-dependence of $R$ implies that $d \mathcal{M} / d p=0$.

In terms of the level density parameter $a=\pi^{2} m^{*} /\left(2 p_{F}^{2}\right)$, and

$$
\begin{aligned}
& p_{F}=\left(\frac{2 n}{C_{2}}\right)^{1 / 2}, \quad Q=1-\frac{n}{m^{*}} \frac{d m^{*}}{d n} \\
& L_{F}=p_{F}^{2} \frac{\mathcal{M}_{F}^{\prime 2}}{m^{* 2}}+p_{F}^{2} \frac{\mathcal{M}_{F}^{\prime \prime}}{m^{*}}-p_{F} \frac{\mathcal{M}_{F}^{\prime}}{m^{*}}
\end{aligned}
$$

Eq. 49p leads to

$$
\begin{aligned}
S & =\frac{4}{3} a T+\frac{56}{45 \pi^{2}} a^{3} T^{3} L_{F}, \quad E_{t h}=\frac{2}{3} a T^{2}+\frac{14}{15 \pi^{2}} a^{3} T^{4} L_{F} \\
P_{t h} & =\frac{2}{3} a n Q T^{2}+\frac{14}{15 \pi^{2}} a^{3} n Q T^{4}\left(L_{F}-\frac{n}{3 Q} \frac{d L_{F}}{d n}\right) \\
\mu_{t h} & =-\frac{2}{3} a T^{2}(1-Q)-\frac{14}{45 \pi^{2}} a^{3} T^{4}\left[L_{F}(1-3 Q)+n \frac{d L_{F}}{d n}\right] \\
C_{V} & =\frac{4}{3} a T+\frac{56}{15 \pi^{2}} a^{3} T^{3} L_{F} \quad \text { and } \quad C_{P}=C_{V}+\frac{16}{9} \frac{a^{2} Q^{2} T^{3}}{\frac{d P_{0}}{d n}} .
\end{aligned}
$$

The above results do not include the effects of collective excitations near the Fermi surface or of non-analytic contributions. As pointed out in Ref. 10, 2-dimensional Fermi systems in condensed matter physics (even with contact interactions) have $T^{2}$ contributions to the entropy from interactions separate from those due to the collective modes. These $T^{2}$ contributions arise from nonanalytic corrections to the real part of the self-energy.

\section{APPLICATION TO MODELS}

In what follows, we compare the analytical results from the leading order corrections to Landau Fermi-liquid theory to the results of exact numerical calculations of the thermal state variables. These comparisons are made using models that are widely used in nuclear and neutron star phenomenology. In the category of non-relativistic potential models, we begin with the model, referred to as $\operatorname{MDI}(\mathrm{A})$, that reproduces the empirical properties of isospin symmetric and asymmetric bulk nuclear matter [13, optical model fits to nucleon-nucleus scattering data [14, heavy-ion flow data in the energy range $0.5-2 \mathrm{GeV} / \mathrm{A}$ [15], and the largest well-measured neutron star mass of $2 \mathrm{M}_{\odot}$ [16, 17. This model, which is based on Refs. [18, 19, incorporates finite range interactions through a Yukawa-type, finite-range force, is contrasted with a conventional zero-range Skyrme model known as $\mathrm{SkO}^{\prime}$ 20. Both models predict nearly identical zerotemperature properties at all densities and proton fractions, including the neutron star maximum mass, but differ in their predictions for heavy-ion flow data 
[21. To provide a contrast, we also investigate a relativistic mean-field theoretical (MFT) model [13] which yields zero-temperature properties similar to those of the two non-relativistic models chosen here. For all three models, we consider nucleonic matter in its pure neutron-matter (PNM, with $\gamma=2)$ and symmetric nuclear matter (SNM, with $\gamma=4$ ) configurations.

\subsection{Finite-range potential models}

For the MDI(A) model [19, 13, the momentum-dependent part of the singleparticle potential is given by

$$
\begin{aligned}
R(p) & =\frac{2 C_{\gamma}}{n_{0}} \frac{2}{(2 \pi \hbar)^{3}} \int d^{3} p^{\prime} f_{p^{\prime}} \frac{1}{1+\left(\frac{\vec{p}-\vec{p}^{\prime}}{\Lambda}\right)^{2}} \\
& \stackrel{T=0}{\longrightarrow} \frac{C_{\gamma}}{n_{0}} \frac{\Lambda^{3}}{\pi^{2} \hbar^{3}}\left\{\frac{p_{F}}{\Lambda}-\arctan \left(\frac{p+p_{F}}{\Lambda}\right)+\arctan \left(\frac{p-p_{F}}{\Lambda}\right)\right. \\
& \left.+\frac{\left(\Lambda^{2}+p_{F}^{2}-p^{2}\right)}{4 \Lambda p} \ln \left[\frac{\Lambda^{2}+\left(p+p_{F}\right)^{2}}{\Lambda^{2}+\left(p-p_{F}\right)^{2}}\right]\right\} .
\end{aligned}
$$

For the coefficients $n_{0}, C_{2}, C_{4}$ and $\Lambda$ we use the values $0.16 \mathrm{fm}^{-3},-23.06 \mathrm{MeV}$, of $R(p)$ and their connection with $\mathcal{M}$ and $L_{F}$ are provided in Appendix A. The MDI Hamiltonian density is shown in Appendix B. For details of the exact numerical calculations, see Ref. 13.

\subsection{Zero-range Skyrme models}

Zero-range Skyrme models belong to that subset of the $D=3$ case for which $L_{F}=0$. This is because, for these models, the momentum-dependent part of the potential arising from the exchange part of zero-range $p$-wave interactions has the form (cf. Appendix C)

$$
R=\frac{p^{2}}{2 m} \beta n
$$

( $\beta$ being a strength constant) which renders the generalized effective mass func${ }_{230}$ tion to be $p$-independent:

$$
\mathcal{M}=\frac{m}{1+\frac{m}{p} \frac{d R}{d p}}=\frac{1}{1+\beta n},
$$

and therefore its derivatives $\mathcal{M}^{\prime}=\mathcal{M}^{\prime \prime}=0$. Hence $L_{F}=0$ as well. Consequently, the results in Sec. 3 for Skyrme models simplify considerably. Results to be shown here are for the $\mathrm{SKO}^{\prime}$ model 20, the exact numerical calculations for which are described in Ref. [13]. 


\subsection{Relativistic models}

The single-particle energy spectrum of relativistic mean-field theoretical models 22] obtained from the nucleon equation of motion has the structure

$$
\epsilon=E^{*}+U(n), \quad E^{*}=\left[p^{2}+M^{* 2}(n, T)\right]^{1 / 2} .
$$

The single-particle potential $U(n)$ is the result of vector meson exchanges whereas the Dirac effective mass $M^{*}$ arises from scalar meson interactions. The implementation of the above equations in the Sommerfeld expansion is made possible by the identification

$$
y=\frac{E^{*}}{T}, \quad \frac{d y}{d p}=\frac{p}{E^{*} T} \quad \text { and } \quad \phi(y)=\frac{E^{*} T}{p} g(p) .
$$

The calculation of $d \phi / d y, d^{3} \phi / d y^{3}$ and $n$ proceeds as in the non-relativistic case with the replacement $\mathcal{M} \rightarrow E^{*}$ [cf. Eq. (8)]. In particular, for $p_{\mu}$ we have

$$
p_{\mu}=p_{F}\left[1-\frac{\pi^{2}}{6} \frac{E_{F}^{* 2} T^{2}}{p_{F}^{4}}\left(D-2+\frac{p_{F} E_{F}^{* \prime}}{E_{F}^{*}}\right)\right]
$$

where

$$
E_{F}^{*}=\left(p_{F}^{2}+M^{* 2}\right)^{1 / 2} \quad \text { and } \quad E_{F}^{* \prime}=\left.\frac{d E^{*}}{d p}\right|_{p_{F}}=\frac{p_{F}}{E_{F}^{*}} .
$$

The simple dependence of $E^{*}$ on the momentum $p$ in Eq. (60) leads to the correspondingly straightforward expression 63 for $E_{F}^{* \prime}$ which, as we will show soon hereafter, results in an elementary form for $L_{F}$ and by extension the whole set of the MFT thermodynamics can be written in an uncomplicated manner.

Substituting Eq. (63) into Eq. 62) yields

$$
p_{\mu}=p_{F}\left[1-\frac{\pi^{2}}{6} \frac{E_{F}^{* 2} T^{2}}{p_{F}^{4}}\left(D-2+\frac{p_{F}^{2}}{E_{F}^{* 2}}\right)\right] .
$$

The twice-by-parts integration of Eq. 17) for the entropy density in the relativistic context gives

$$
s=\frac{C_{D}}{T} \int d p p^{D-1} f_{p}\left(E^{*}+\frac{p}{D} \frac{d E^{*}}{d p}-E_{\mu}^{*}\right)
$$

where one observes the analogy with the integral term of Eq. 20. Using

$$
g_{s}(p)=p^{D-1}\left(E^{*}+\frac{p}{D} \frac{d E^{*}}{d p}-E_{\mu}^{*}\right)
$$

we proceed as before to get the entropy density in terms of $p_{\mu}$ as

$$
s=\frac{\pi^{2}}{3} C_{D} p_{\mu}^{D-2} E_{\mu}^{*} T+\frac{7 \pi^{2}}{90} C_{D}(D-2)(D-4) p_{\mu}^{D-6} E_{\mu}^{* 3} T^{3}\left[1+\frac{3}{(D-4)} \frac{p_{\mu}^{2}}{E_{\mu}^{* 2}}\right]
$$


which, with the aid of Eq. 62, becomes

$$
\begin{aligned}
s & =\frac{\pi^{2}}{3} C_{D} p_{F}^{D-2} E_{F}^{*} T+\frac{\pi^{4}}{45} C_{D}(D-2)(D-9) p_{F}^{D-6} E_{F}^{* 3} T^{3} \\
& \times\left[1+\frac{11}{2(D-9)} \frac{p_{F}^{2}}{E_{F}^{* 2}}-\frac{5}{2(D-2)(D-9)} \frac{p_{F}^{4}}{E_{F}^{* 4}}\right] .
\end{aligned}
$$

255 In the derivation of the last equation the weak temperature of $M^{*}$ in the degenerate limit has been ignored (but not of $E_{\mu}^{*}$ ). Combining Eq. (68) with Eqs. 25D-27) in $D=3$, and using the definitions [here, the Fermi temperature $\left.T_{F}=p_{F}^{2} /\left(2 E_{F}^{*}\right)\right]$

$$
a=\frac{\pi^{2}}{2} \frac{E_{F}^{*}}{p_{F}^{2}}, \quad q=\frac{M^{* 2}}{E_{F}^{* 2}}\left(1-\frac{3 n}{M^{*}} \frac{d M^{*}}{d n}\right) \quad \text { and } \quad L_{F}=\frac{11}{12} \frac{p_{F}^{2}}{E_{F}^{* 2}}-\frac{5}{12} \frac{p_{F}^{4}}{E_{F}^{* 4}},
$$

we obtain

$$
\begin{aligned}
S & =2 a T-\frac{16}{5 \pi^{2}} a^{3} T^{3}\left(1-L_{F}\right), \quad E_{t h}=a T^{2}-\frac{12}{5 \pi^{2}} a^{3} T^{4}\left(1-L_{F}\right) \\
P_{t h} & =\frac{1}{3} a n T^{2}(1+q)-\frac{4}{5 \pi^{2}} a^{3} n T^{4}\left[1-L_{F}+q\left(1-\frac{L_{F}}{3}-\frac{10}{9} \frac{p_{F}^{4}}{E_{F}^{* 4}}\right)\right] \\
\mu_{t h} & =-\frac{2}{3} a T^{2}\left(1-\frac{q}{2}\right)-\frac{4}{5 \pi^{2}} a^{3} T^{4} q\left(1-\frac{L_{F}}{3}-\frac{10}{9} \frac{p_{F}^{4}}{E_{F}^{* 4}}\right) \\
C_{V} & =2 a T-\frac{48}{5 \pi^{2}} a^{3} T^{3}\left(1-L_{F}\right) \quad \text { and } \quad C_{P}=C_{V}+\frac{4}{9} \frac{a^{2} T^{3}(1+q)^{2}}{\frac{d P_{0}}{d n}}
\end{aligned}
$$

As in the non-relativistic case, when conditions are such that $d P_{0} / d n$ is small, one must use derivatives of the pressure with respect to $n$ and $T$ that include thermal contributions to $\mathcal{O}\left(T^{4}\right)$ in the calculation of $C_{P}$. Explicitly,

$$
\begin{aligned}
\left(\left.\frac{\partial P_{t h}}{\partial T}\right|_{n}\right)^{2} & =\frac{4}{9} a^{2} n^{2} T^{2}(1+q)^{2} \\
& \times\left\{1-\frac{48}{5 \pi^{2}} \frac{a^{2} T^{2}}{1+q}\left[1-L_{F}+q\left(1-\frac{L_{F}}{3}-\frac{10}{9} \frac{p_{F}^{4}}{E_{F}^{* 4}}\right)\right]\right\} \quad(74) \\
\left.\frac{\partial P}{\partial n}\right|_{T} & =\frac{d P_{0}}{d n}+\frac{T^{2} a}{9}\left[(1+q)(2-q)+3 n \frac{d q}{d n}\right] \\
& +\frac{4}{5 \pi^{2}} T^{4} a^{3} q\left[1-L_{F}+\left(q-\frac{n}{q} \frac{d q}{d n}\right)\left(1-\frac{L_{F}}{3}-\frac{10}{9} \frac{p_{F}^{4}}{E_{F}^{* 4}}\right)\right. \\
& \left.+\frac{n}{q} \frac{d L_{F}}{d n}\left(1+\frac{q}{3}\right)+\frac{40}{27} \frac{p_{F}^{4}}{E_{F}^{* 4}} q\right] \\
\frac{d q}{d n} & \left.=-\frac{2}{3 n} q(1-q)+\frac{1}{M^{*}} \frac{d M^{*}}{d n} q-\frac{M^{*}}{2 E_{F}^{* 2}}\left(\frac{d M^{*}}{d n}+\frac{3 n}{2} \frac{d^{2} M^{*}}{d n^{2}}\right) 7.6\right)
\end{aligned}
$$




\subsection{Relativistic models beyond the Hartree mean-field theory}

Contributions from two-loops that account for exchange contributions from meson-mediated interactions between nucleons alter the structure of the singleparticle spectrum $\epsilon$ from its form in MFT. Including such contributions in terms of a momentum-dependent self-energy, $\epsilon$ acquires the general structure

$$
\epsilon=E^{*}+R(n, p)+U(n) \equiv \Sigma(n, p)+U(n)
$$

which suggests the identifications

$$
y=\frac{\epsilon-U(n)}{T}=\frac{\Sigma}{T}, \quad \frac{d y}{d p}=\frac{p}{\mathcal{M} T} \quad \text { and } \quad \phi(y)=\frac{\mathcal{M} T}{p} g(p)
$$

for carrying out the Sommerfeld expansion. These expressions are formally the

same as Eqs. (6) and (7) in the non-relativistic case with the difference that the effective mass function is now defined as

$$
\mathcal{M}=E^{*}\left(1+\frac{E^{*}}{p} \frac{d R}{d p}\right)^{-1}=p\left(\frac{d \Sigma}{d p}\right)^{-1}
$$

with its first and second derivatives with respect to $p$ given by

$$
\begin{aligned}
\mathcal{M}^{\prime} & =\frac{\mathcal{M}}{p}\left(1-\mathcal{M} \frac{d^{2} \Sigma}{d p^{2}}\right) \\
\mathcal{M}^{\prime \prime} & =\mathcal{M}^{\prime}\left(\frac{\mathcal{M}^{\prime}}{\mathcal{M}}-\frac{1}{p}\right)-\frac{\mathcal{M} \mathcal{M}^{\prime}}{p}\left(\frac{d^{2} \Sigma}{d p^{2}}+\frac{\mathcal{M}}{\mathcal{M}^{\prime}} \frac{d^{3} \Sigma}{d p^{3}}\right) .
\end{aligned}
$$

The twice-by-parts integration of the expression for the entropy density yields

$$
s=\frac{C_{D}}{T} \int d p p^{D-1} f_{p}\left(\Sigma+\frac{p}{D} \frac{d \Sigma}{d p}-\Sigma_{\mu}\right) .
$$

The use of Maxwell's relations described in earlier sections leads to results that are identical to Eqs. $(23)$ and $(24)$ for the $D$-dimensional case, and Eqs. (28)(47) in the $D=3$ case, but with the effective mass function in Eq. (79). As in the non-relativistic case, we have ignored the $T$-dependence of $\mathcal{M}_{\mu}$ in the first term of the entropy density in going from Eq. (23) to Eq. (24). In principle, $\mathcal{M}_{\mu}=\mathcal{M}_{F}+\mathcal{O}\left(T^{2}\right)$ but we have chosen to ignore the $T$-dependence of $\mathcal{M}$ as befits a degenerate-limit analysis.

\section{LEPTONS AND PHOTONS}

In physical applications involving neutron stars and supernovae, contributions from leptons (electrons, positrons, and muons) and photons must be included to all of the state variables. Thermal effects from these sources are adequately given by their ideal gas forms. This is because contributions from electromagnetic interactions are small owing to the smallness of the fine-structure constant $\alpha=e^{2} /\left(4 \pi \epsilon_{0} \hbar c\right) \simeq 1 / 137$. The leading contributions arise from a 
two-loop diagram involving photon exchange (referred to as the exchange term because in the $T=0$ limit it arises from a pair of fermions exchanging their three-momenta in the Fermi sea). Compared to their non-interacting counterparts, limiting forms of the exchange contribution to the pressure highlight the sub-leading role of interactions [23]:

$$
\begin{aligned}
P_{\mathrm{ex}} & =-\frac{\alpha}{4 \pi^{3}}\left\{\frac{3}{2}\left[\mu p_{F}-m^{2} \ln \left(\frac{\mu+p_{F}}{m}\right)\right]^{2}-p_{F}^{4}\right\} \quad(\text { for } T=0) \\
& =-\frac{\pi \alpha}{72}\left(5 T^{4}+\frac{18}{\pi^{2}} \mu^{2} T^{2}+\frac{9}{\pi^{4}} \mu^{4}\right) \quad(\text { for } m=0) \\
& =-\frac{\alpha}{8 \pi^{2}} m^{2} T^{2} e^{2(\mu-m) / T} \quad(\text { for } T \ll m-\mu \ll m ; \text { classical limit })
\end{aligned}
$$

where the Fermi momentum $p_{F}=\left(\mu^{2}-m^{2}\right)^{1 / 2}$ when $|\mu|>m$. The first-order correction to the pressure in the limit $\mu \gg m_{e}, T$ (in $\hbar=c=1$ units) renders the total pressure as

$$
P=P_{i d}+P_{e x}=\frac{\mu^{4}}{12 \pi^{2}}\left(1-\frac{3}{2} \frac{\alpha}{\pi}\right) .
$$

As demonstrated in Ref. 223 (see Chap. 5.6), the Chandrasekhar limit for a white dwarf is changed by only $0.2 \%$ (likely not discernible by measuring white dwarf masses) with the inclusion of photon-exchange contributions. The classical-limit result in Eq. 83) modifies the ideal gas EOS to

$$
P=P_{i d}+P_{e x}=n T\left(1-\frac{\pi}{2} \alpha \frac{n}{m T^{2}}\right),
$$
tions from electromagnetic interactions are generally ignored in homogeneous dense matter although they are easily incorporated in calculations (cf. Eq. (5.55) in Ref. 23). In the results to be presented below, we have adapted the numerical methods devised in Ref. 24] for the calculation of the ideal-gas leptonic contributions for arbitrary degeneracy. Contributions from photons (significant only at high values of $T$ and $n$ ) are given by their familiar forms

$$
\varepsilon_{\gamma}=\frac{\pi^{2}}{45} \frac{T^{4}}{(\hbar c)^{3}}, \quad P_{\gamma}=\frac{\varepsilon_{\gamma}}{3}, \quad \text { and } \quad s_{\gamma}=\frac{4}{3} \frac{\varepsilon_{\gamma}}{T} .
$$

The influence of thermal effects from leptons and photons to the total has been detailed in Ref. 13, and will not be repeated here.

\section{RESULTS AND DISCUSSION}

Here, we compare the results from FLT and FLT+NLO with the exact numerical results for the two non-relativistic models $\left(\mathrm{MDI}(\mathrm{A})\right.$ and $\left.\mathrm{SkO}^{\prime}\right)$, and for 
a relativistic mean-field theoretical model (MFT). Numerical techniques for obtaining the exact numerical results are detailed in Refs. [24, 25]. The thermal properties presented for PNM and SNM are at a temperature of $T=20 \mathrm{MeV}$.

In the top panels of Fig. 1 the Landau effective masses $m_{n}^{*}$ of the neutron scaled with its vacuum value are shown as a function of baryon density $n$. For the MFT model, both $m_{n}^{*}=E_{F_{n}}^{*}=\sqrt{M^{*^{2}}+p_{F_{n}}^{2}}$ and the Dirac effective mass $M^{*}$ are shown. Noteworthy points for the non-relativistic models are: (i) The for SNM - although quantitative differences are present, and (ii) except for $n$ up to $0.2 \mathrm{fm}^{-3}$, the decrease with increasing $n$ for the MDI model is relatively slow (logarithmic decline) compared with that for the $\mathrm{SkO}^{\prime}$ model $\left[(1+\beta n)^{-1}\right.$ fall off]. This overall flatness of $m^{*}$ for the MDI model is a direct consequence of the momentum structure of its single-particle potential which causes it to saturate at high momenta. For the MFT model, $M^{*}$ decreases monotonically with $n$ to values lower than those for the non-relativistic models. The Landau mass $m^{*}$, however, exhibits a non-monotonic behavior, attaining a minimum for $n_{\min }=0.57(0.52) \mathrm{fm}^{-3}$ obtained from the solution of

$$
\frac{p_{F}}{M^{*}}+\frac{d M^{*}}{d p_{F}}=0
$$

for the case of SNM (PNM), and increasing thereafter due to the monotonic increase of $p_{F_{n}}$ with $n$. Physically, $n_{\min }$ marks the transition of nucleons well into the relativistic region. The density $n_{R}$ at which $p_{F}=M^{*}$ occurs at

$$
n_{R}=0.643 \gamma\left(\frac{m^{*}}{m}\right)^{3} \mathrm{fm}^{-3}
$$

about $(2 / 3) n_{\min }=0.38(0.34) \mathrm{fm}^{-3}$ for SNM (PNM) which signals the onset of relativistic effects which become progressively important for $n \geq n_{R}$.

The bottom panels of Fig. 1 show the logarithmic derivatives of $m_{n}^{*}$ vs $n$. Also shown for the MFT model is $d \ln M^{*} / d \ln n$ which has been divided by a factor of 3 to fit within the figure. The logarithmic derivatives $m^{*}$ for MDI(A) show little variation with $n$ at supra-nuclear densities. In contrast, results for the with $n$. The logarithmic derivative of $M^{*}$ in MFT drops to values considerably lower than for the other two models. This derivative remains negative but approaches a constant value at large densities. The logarithmic derivative of the Landau effective mass drops until $\sim 2 n_{0}$, but then increases to positive values.

345 This behavior is a reflection of the minimum that occurs for $m_{n}^{*}$ in this model. As will be seen below, the density dependences of the effective masses and their logarithmic derivatives determine the behavior of all the thermal properties in FLT. Higher order derivatives of the Landau effective mass function in Eq. (8) appear in FLT+NLO. 
The FLT and FLT+NLO results for the thermal energy, $E_{t h}$ vs $n$, are compared with the exact numerical results in Fig. 2, The NLO corrections to FLT yield agreement with the exact results down to sub-nuclear densities of 0.5 to 1 $n_{0}$ compared to 2-3 $n_{0}$ for FLT. As is the case with FLT, slightly but systematically better agreement with FLT+NLO occurs for PNM than for SNM for all the thermodynamic quantities we study. This is a consequence of the fact that the neutron density in PNM $\left(n_{n}=n\right)$ is twice the neutron density in SNM $\left(n_{n}=n / 2\right)$; PNM is more degenerate than SNM at the same baryon density $n$.

In Figure 3, we show the convergence of FLT and FLT+NLO results to the exact numerical results for $P_{t h}$. For all three models, the FLT+NLO results extend the agreement with the exact results to lower densities than those of FLT. The SkO' model shows the greatest changes relative to FLT for both SNM and PNM. Both the MDI(A) and MFT results also show improvement using FLT+NLO with better agreement occurring for PNM. For $P_{t h}$ at $n>n_{0}$, the influence of $m^{*}(n)$ and its logarithmic derivative with respect to $n$ (in FLT), and the higher derivatives of $\mathcal{M}$ (in FLT+NLO) are amply demonstrated: (i) for $\operatorname{MDI}(\mathrm{A}), P_{t h}$ grows very slowly with $n$; (ii) considerably larger growth with $n$ is exhibited for $\mathrm{SkO}^{\prime}$ than for $\mathrm{MDI}(\mathrm{A})$; and (iii) for MFT, the prominent peaks 370 in $P_{t h}$ at intermediate densities are due to the minima in $m^{*}$ and $d \ln M * / d \ln n$ at similar densities. For asymptotic densities, $P_{t h} \propto n^{4 / 3}$ in MFT models characteristic of massless particles. A corresponding behavior is also present in the thermal chemical potentials (see below).

375 Comparisons of the entropy per baryon, $S$, are shown in Fig. 4. For all three models, the agreement between the exact results and those of FLT+NLO extends to below $n_{0}$, and to as low as $n_{0} / 4$ for PNM with MFT. This is an improvement from the FLT results for which convergence ranged from $1.5 n_{0}$ (FLT and PNM) to $3 n_{0}\left(\mathrm{SkO}^{\prime}\right.$ and $\left.\mathrm{SNM}\right)$.

In Fig. 5, the thermal parts of the neutron chemical potentials, $\mu_{n, t h}$, are shown as a function of $n$. Results from FLT + NLO lie closer to the exact results than do those of FLT with the agreement extending to sub-nuclear densities. As with the other state variables, the agreement is quantitatively better for PNM than for SNM.

The specific heat at constant volume, $C_{V}$, is presented in Fig. 6 as a function of $n$. For all models, the FLT+NLO results show better agreement with the exact results than those of FLT. The lowest density for which the agreement 390 extends differs between the models, that for the $\mathrm{SkO}^{\prime}$ model being higher than for the other two models particularly for SNM.

In Figure 7 we show the specific heat at constant pressure and its limiting cases as functions of $n$. The maxima at low sub-nuclear densities in the exact numerical results are related to the liquid-gas phase transition of nucleonic matter which occurs at $T \sim 15-20 \mathrm{MeV}$. The convergence between the exact 
and approximate results for MDI(A) and MFT follows a pattern similar to the quantities discussed previously with the conspicuous exception of FLT which appears to outperform FLT+NLO for SNM. We attribute this feature to a numerical accident, possibly due to the proximity to the phase transition. Note that FLT begins to deviate from the exact result for densities below $n \sim 3 n_{0}$ in the case of $\mathrm{SkO}^{\prime}$. As our analysis here is concerned with the degenerate region, this failure at sub-nuclear densities is not surprising. For an adequate treatment in the non-degenerate region, see Ref. [13.

405 degenerate to degenerate matter can be described to next-to-leading order in
$T / T_{F}$ for models with general momentum dependences in their single-particle potentials. Analytical formulas valid to next-to-leading order in $T / T_{F}$ for all

The thermal index, $\Gamma_{t h}=1+\left(P_{t h} / \varepsilon_{t h}\right)$, is shown in Fig. 8 . For the $\mathrm{SkO}^{\prime}$ model, the FLT result is exact for all regions of degeneracy. This happenstance is due to the fact that for non-relativistic nucleons with only contact interactions, $P_{t h}$ and $\varepsilon_{t h}$ can be written entirely in terms of their ideal-gas counterparts as

$$
\begin{aligned}
& P_{t h}(n, T)=P_{t h}^{i d}\left(n, T ; m^{*}\right) Q \\
& \varepsilon_{t h}(n, T)=\varepsilon_{t h}^{i d}\left(n, T ; m^{*}\right), \quad \frac{P_{t h}^{i d}}{\varepsilon_{t h}^{i d}}=\frac{2}{3}
\end{aligned}
$$

regardless of the degree of degeneracy. The $n$-dependence of $m^{*}$ for Skyrme-like interactions thus yields

$$
\Gamma_{t h}=\frac{8}{3}-\frac{m^{*}(n)}{m} .
$$

For the finite-range MDI(A) and the relativistic MFT models, the FLT+NLO results mildly improve the FLT results in reproducing the exact ones. This marginal improvement is related to the ratio $P_{t h} / \varepsilon_{t h}$ in $\Gamma_{t h}$ in these cases, 415 which amount to a constant plus a correction due to effects of $n$ plus a second correction due to $(n, T)$ effects, which means that temperature effects are subleading, and therefore very weak. Note that the results for the three models differ significantly from each other, both qualitatively and quantitatively. These differences are due to the differences in the effective mass functions of the models.

\section{SUMMARY AND CONCLUSIONS}

For homogeneous systems of fermions in the limit of extreme degeneracy $\left(T / T_{F} \ll 1\right.$, where $T_{F}$ is the Fermi temperature), Landau's Fermi Liquid Theory (FLT) provides simple analytical expressions that are model independent for the thermal state variables (e.g., entropy, energy, pressure, chemical potential, and specific heats) [1. In the absence of collective excitations close to the Fermi surface, thermal effects are primarily determined by the nucleon's Landau effective mass and its first density derivative which in turn depend on the momentum-dependence of the $T=0$ single-particle energy spectrum.

In this work, we have developed a method by which thermal effects in neardegenerate to degenerate matter can be described to next-to-leading order in
$T / T_{F}$ for models with general momentum dependences in their single-particle potentials. Analytical formulas valid to next-to-leading order in $T / T_{F}$ for all 
of the thermal state variables are presented. The entropy density and specific heats are carried to $\mathcal{O}\left(T / T_{F}\right)^{3}$ whereas the energy density and pressure to $\mathcal{O}\left(T / T_{F}\right)^{4}$, extending the leading order results of FLT. These extensions involved the use of a generalized Landau effective mass function which enables the calculation of the entropy density, and thereafter the other state variables, for a general single-particle spectrum. In special cases, e.g., models with contact interactions, knowledge of the Landau effective mass suffices. In the case

440 of finite-range interactions, momentum derivatives of the Landau effective mass function up to second order are required to satisfy the thermodynamic identity. Our results are valid for potential and field-theoretical models as long as the underlying interactions yield a single-particle spectrum that is weakly dependent on temperature in the degenerate limit.

We find that $\mathcal{O}\left(T^{3}\right)$ corrections in 2-dimensions for non-relativistic models appear only if they include finite-range interactions or, equivalently, if the momentum content of their mean field is something other than quadratic. These contributions supplement the $\mathcal{O}\left(T^{2}\right)$ non-analytic contributions even with zerorange interactions established previously [10].

To illustrate the density region of their applicability, numerical results from the new formulas were compared with those of exact numerical calculations for zero- and finite-range potential models as well as for relativistic mean-field theoretical (MFT) models widely used in astrophysical applications of hot and dense nuclear matter. In all cases, excellent agreement with the exact results ${ }_{455}$ was found even to sub-nuclear densities of $\sim 0.1 \mathrm{fm}^{-3}$ for $T=20 \mathrm{MeV}$, whereas FLT results are valid only for densities beyond $\sim 0.3 \mathrm{fm}^{-3}$. (For low temperatures and below $\sim 0.1 \mathrm{fm}^{-3}$, inhomogeneous phases with nuclei and pasta-like configurations are known to exist, and must be treated separately.) In addition to providing physical insights, our analytical results facilitate a rapid evaluation of the EOS in the homogeneous phase (important for computer-time consuming large-scale simulations of supernovae, proto-neutron stars, and mergers of binary compact objects). Our formulas can be used for any $T=0$ quasi-particle spectrum, e.g., those extracted from Brueckner-Hartree-Fock and Dirac-BruecknerHartree-Fock approaches, extensions of MFT models with non-linear derivatives or with 2-loop effects, and effective field-theoretical approaches.

Examples of contributions not included in our work arise from, e.g., nonanalytic contributions from long-wavelength fluctuations, single particle-hole excitations and, collective and paring correlations close to the Fermi surface. Their roles as functions of densities and temperatures of relevance to astrophysical phenomena need further investigation.

\section{ACKNOWLEDGEMENTS}

This work was supported by the U.S. DOE under Grants No. DE-FG0293ER-40756 and No. DE-FG02-87ER-40317. 


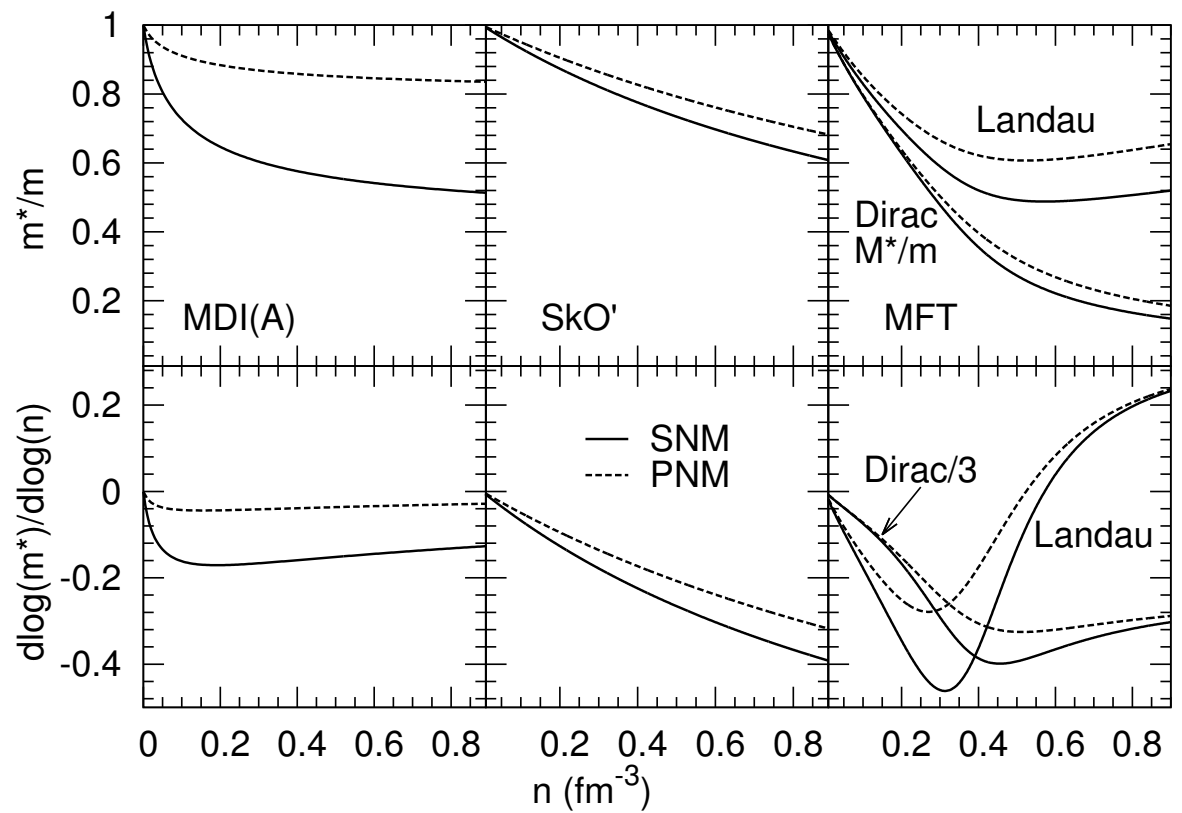

Figure 1: Top panels: Effective mass vs density for non-relativistic potential models (MDI(a) and $\mathrm{SkO}^{\prime}$ ) and relativistic mean-field theoretical model (MFT) for symmetric nuclear matter (SNM) and pure neutron matter (PNM). Bottom panels: Logarithmic derivative of the effective mass with respect to density vs density. 


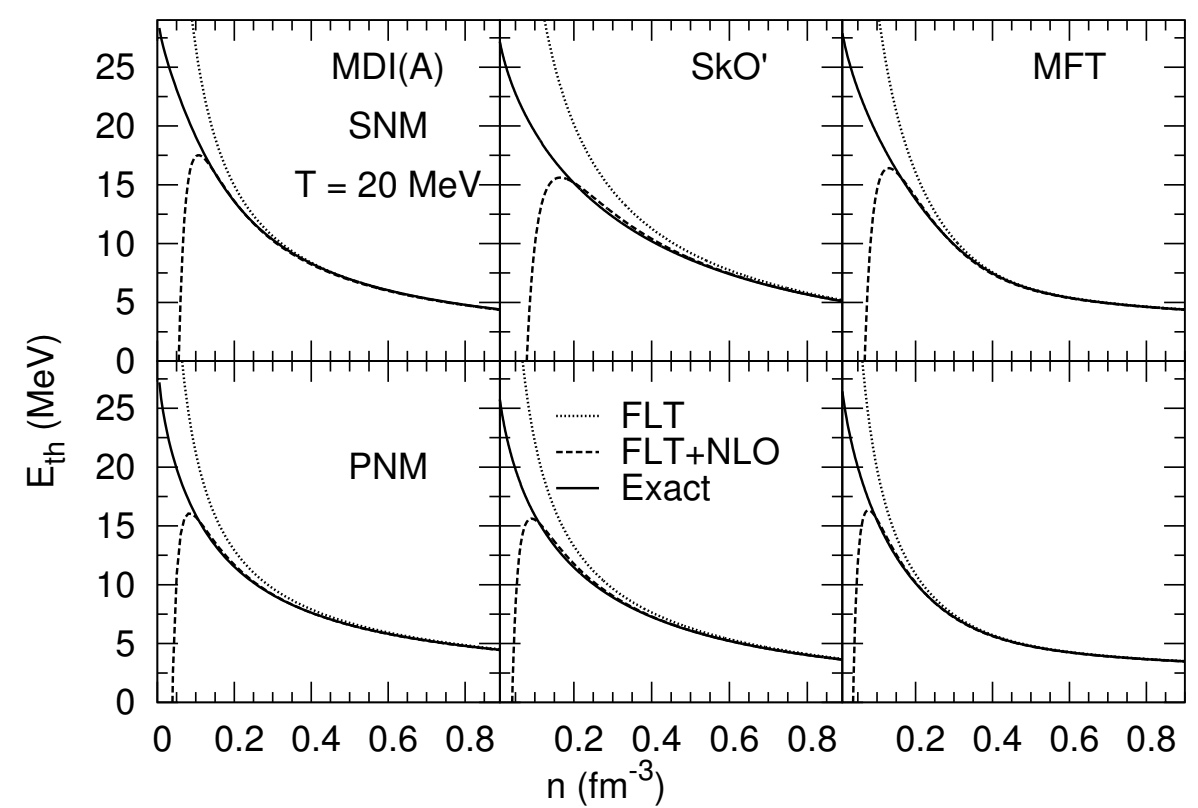

Figure 2: Thermal energy vs baryon density for the three models at a temperature of $T=20$ $\mathrm{MeV}$. Results for SNM are in the top panels and for PNM in the bottom panels.

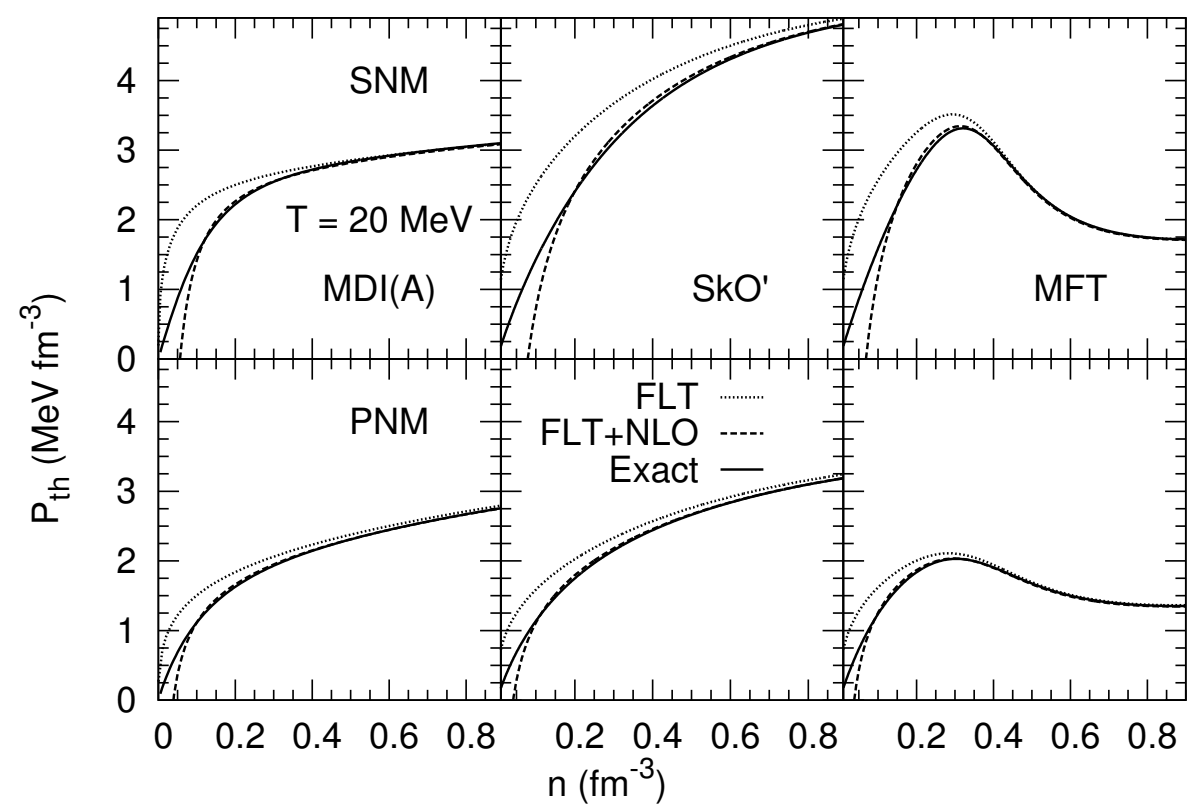

Figure 3: Same as Fig. 2, but for thermal pressure. 


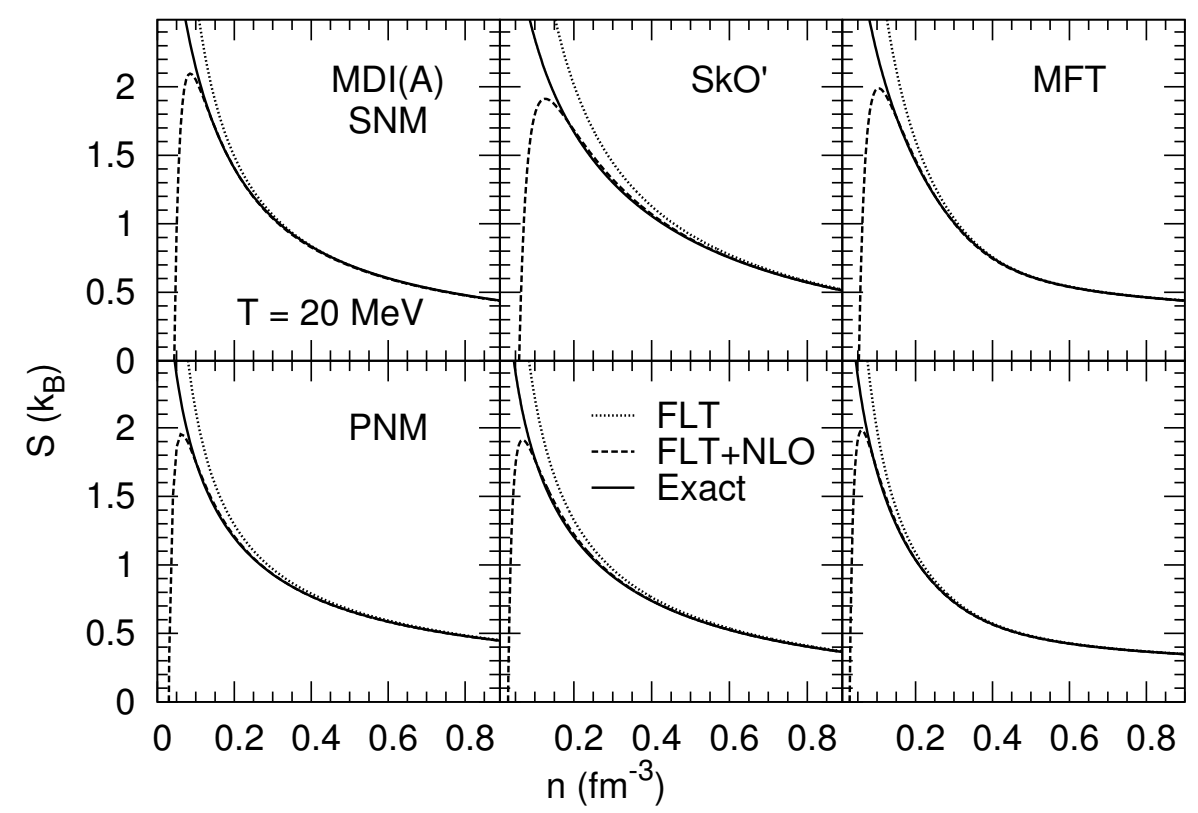

Figure 4: Same as Fig. 2 but for entropy per baryon.

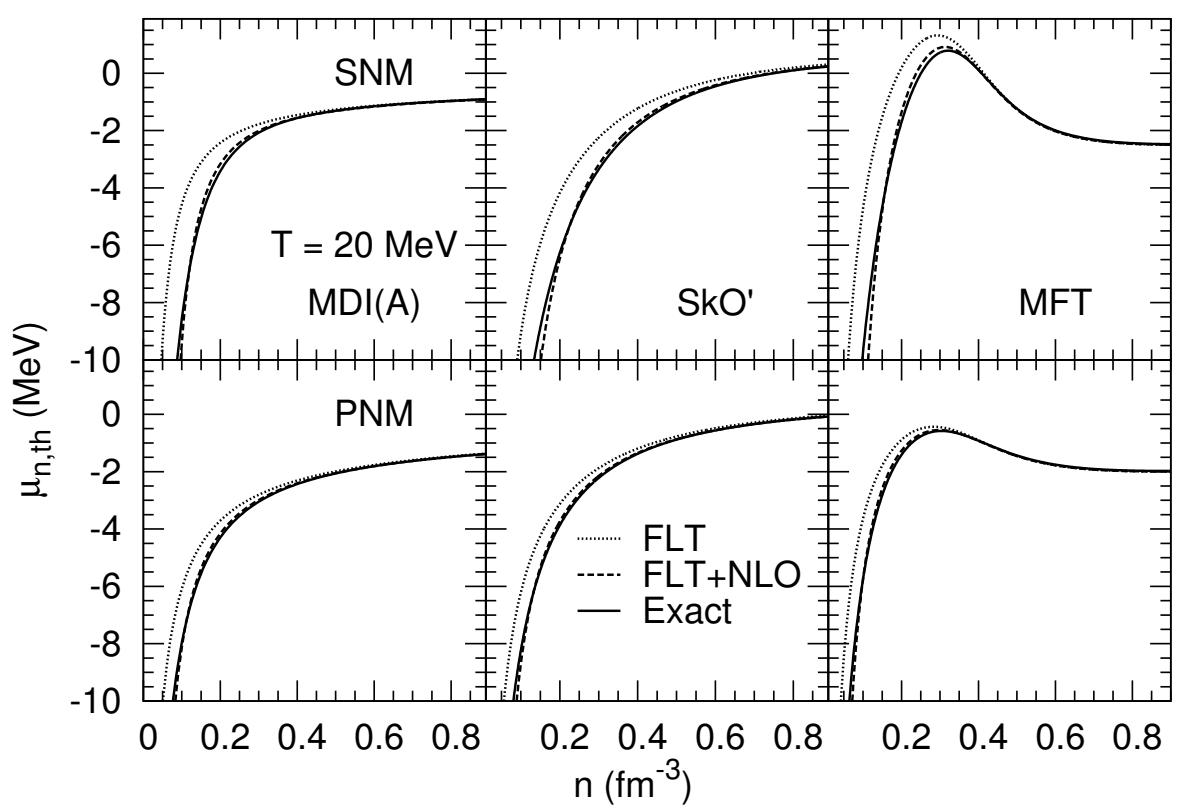

Figure 5: Same as Fig. 2 but for thermal neutron chemical potential. 


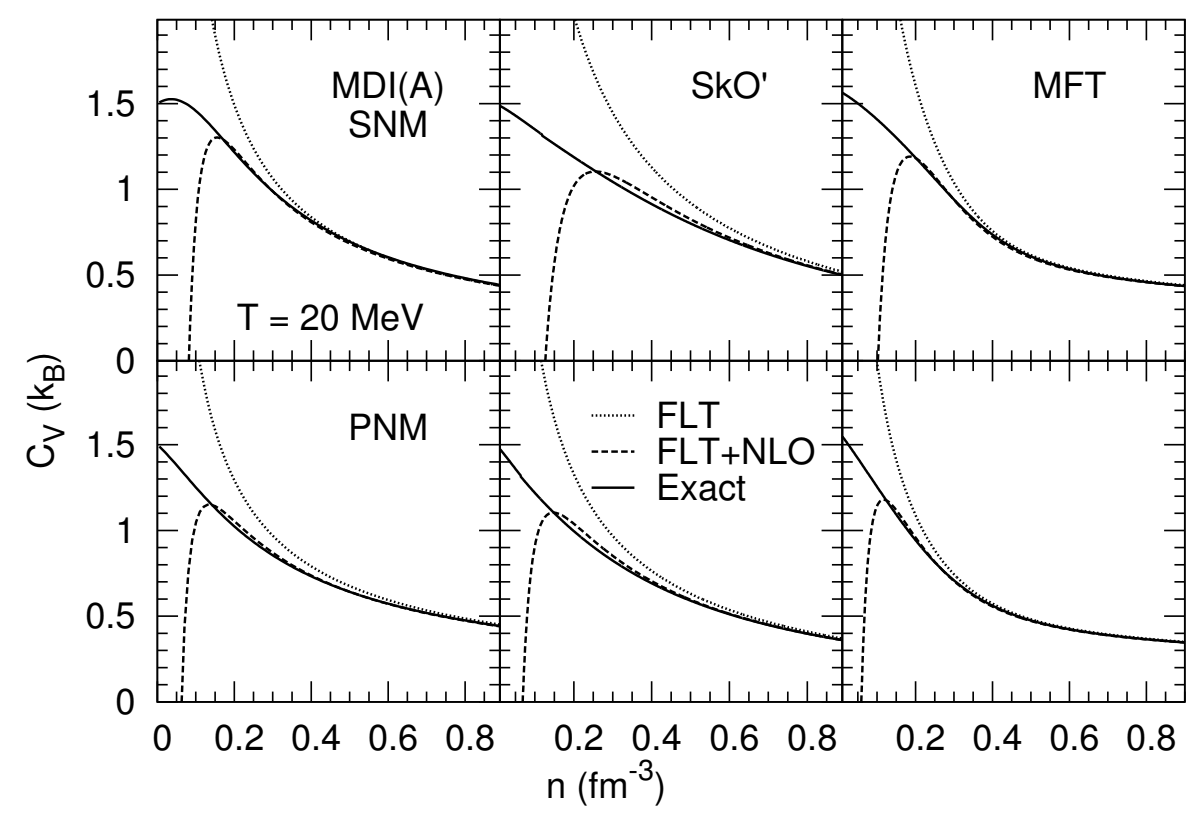

Figure 6: Same as Fig. 2 but for specific heat at constant volume.

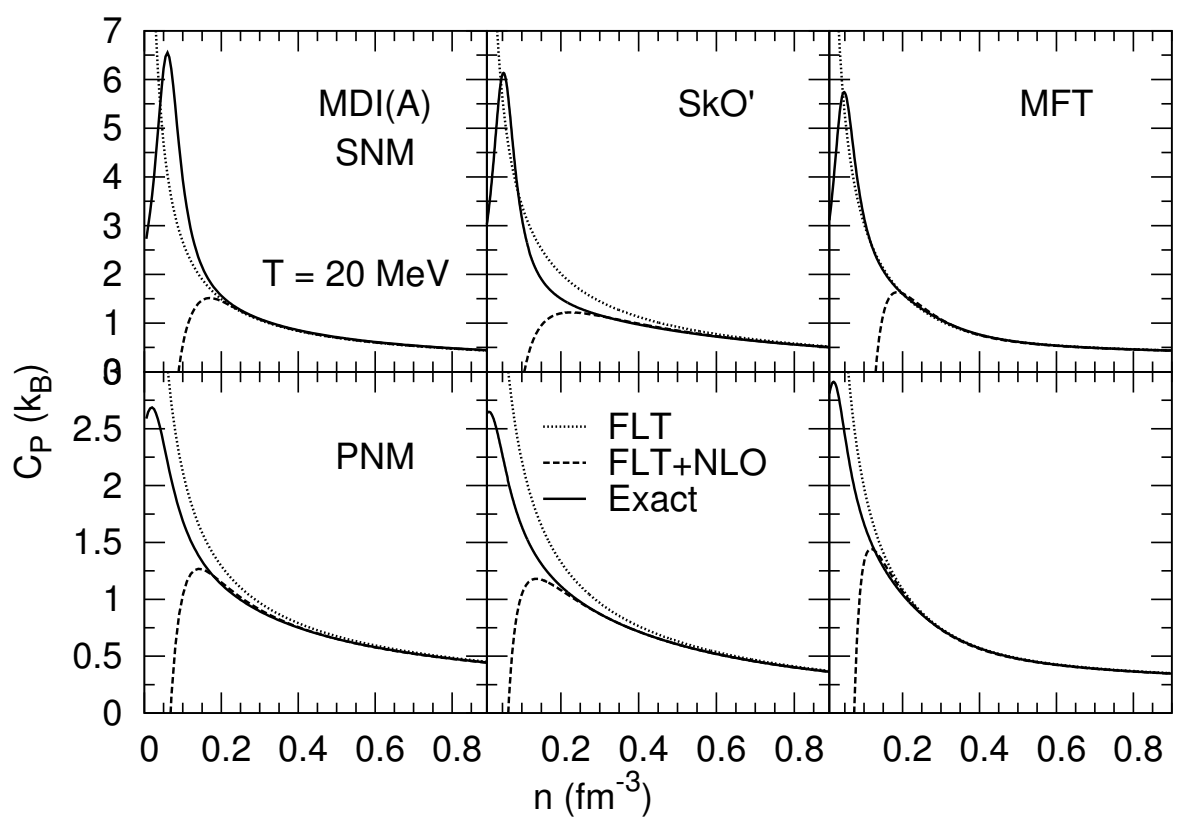

Figure 7: Same as Fig. 2 but for specific heat at constant pressure. 


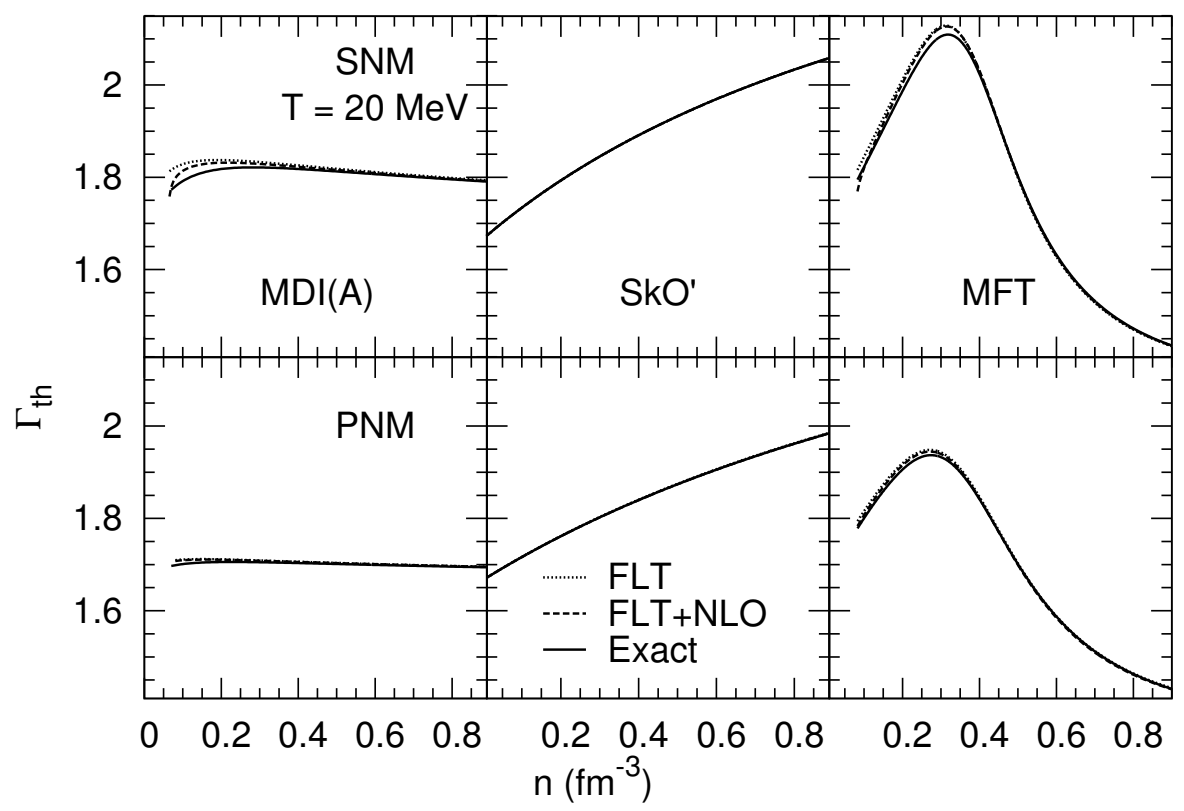

Figure 8: Same as Fig. 2 but for the thermal index. 


\section{Appendix A. RESULTS RELATED TO $L_{F}$}

Here we collect relations required to calculate the quantity $L_{F}$ defined in Eq. 29 of Sec. 3.

Model-independent relations

$$
\begin{aligned}
\mathcal{M}^{\prime} & \equiv \frac{d \mathcal{M}}{d p}=\frac{\mathcal{M}^{2}}{p^{2}}\left(\frac{d R}{d p}-p \frac{d^{2} R}{d p^{2}}\right) \\
\mathcal{M}^{\prime \prime} & =\frac{2 \mathcal{M}^{3}}{p^{4}}\left(\frac{d R}{d p}-p \frac{d^{2} R}{d p^{2}}\right)-\frac{2 \mathcal{M}^{2}}{p^{3}}\left(\frac{d R}{d p}-p \frac{d^{2} R}{d p^{2}}+\frac{p^{2}}{2} \frac{d^{3} R}{d p^{3}}\right) \\
& =-2 \frac{\mathcal{M}^{\prime}}{p}\left(1-\frac{\mathcal{M}}{p}\right)-\frac{\mathcal{M}^{2}}{p} \frac{d^{3} R}{d p^{3}} \equiv \frac{d^{2} \mathcal{M}}{d p^{2}} \\
\frac{d L_{F}}{d n} & =\frac{p_{F}}{3 n} \frac{d L_{F}}{d p_{F}} \\
\frac{d L_{F}}{d p_{F}} & =\frac{5}{12} \frac{\mathcal{M}_{F}^{\prime}}{m^{*}}\left(1-p_{F} \frac{\mathcal{M}_{F}^{\prime}}{m^{*}}\right)\left(1-\frac{42}{5} p_{F} \frac{\mathcal{M}_{F}^{\prime}}{m^{*}}\right) \\
& -\left.\frac{m^{*}}{6} \frac{d^{3} R}{d p^{3}}\right|_{p_{F}}\left(1+\frac{49}{2} p_{F} \frac{\mathcal{M}_{F}^{\prime}}{m^{*}}\right)-\left.\frac{7}{12} p_{F} m^{*} \frac{d}{d p_{F}} \frac{d^{3} R}{d p^{3}}\right|_{p_{F}}
\end{aligned}
$$

The subscript $F$ denotes evaluation at $p=p_{F}$.

MDI model-specific realations

$$
\begin{aligned}
\left.\frac{d R}{d p}\right|_{p_{F}} & =\frac{C_{\gamma}}{n_{0}} \frac{\Lambda^{2}}{\pi^{2} \hbar^{3}}\left[1-\frac{1}{2}\left(1+\frac{\Lambda^{2}}{2 p_{F}^{2}}\right) \ln \left(1+\frac{4 p_{F}^{2}}{\Lambda^{2}}\right)\right] \\
\left.\frac{d^{2} R}{d p^{2}}\right|_{p_{F}} & =-\frac{2 C_{\gamma}}{n_{0}} \frac{\Lambda^{2}}{\pi^{2} \hbar^{3}} \frac{1}{p_{F}\left(4 p_{F}^{2}+\Lambda^{2}\right)}\left[\left(3 p_{F}^{2}+\Lambda^{2}\right)\right. \\
& \left.-\left(p_{F}^{2}+\Lambda^{2}\right)\left(1+\frac{\Lambda^{2}}{4 p_{F}^{2}}\right) \ln \left(1+\frac{4 p_{F}^{2}}{\Lambda^{2}}\right)\right] \\
\left.\frac{d^{3} R}{d p^{3}}\right|_{p_{F}} & =\frac{2 C_{\gamma}}{n_{0}} \frac{1}{\pi^{2} \hbar^{3}} \frac{1}{p_{F}^{2}\left(4 p_{F}^{2}+\Lambda^{2}\right)^{2}} \\
& \times\left[8 p_{F}^{6}+34 p_{F}^{4} \Lambda^{2}+21 p_{F}^{2} \Lambda^{4}+3 \Lambda^{6}\right. \\
\left.\frac{d}{d p_{F}} \frac{d^{3} R}{d p^{3}}\right|_{p_{F}} & \left.=-\frac{3 \Lambda^{2}}{4}\left(1+\frac{\Lambda^{2}}{p_{0}^{2}}\right)\left(4 p_{F}^{2}+\Lambda^{2}\right)^{2} \ln \left(1+\frac{4 p_{F}^{2}}{\Lambda^{2}}\right)\right] \\
& \times\left[4 \hbar_{F}^{2} \frac{1}{p_{F}^{5}\left(4 p_{F}^{2}+\Lambda^{2}\right)^{3}}\right. \\
& -3 \Lambda^{2}\left(p_{F}^{2}+134 p_{F}^{6} \Lambda^{2}+118 p_{F}^{4} \Lambda^{4}+33 p_{F}^{2} \Lambda^{6}+3 \Lambda^{8}\right)
\end{aligned}
$$




\section{Appendix B. MDI HAMILTONIAN DENSITY}

For the MDI models, the Hamiltonian density is composed of terms arising from kinetic sources, $\mathcal{H}_{k}$, density-dependent interactions, $\mathcal{H}_{d}$, and momentumdependent interactions, $\mathcal{H}_{m}$ :

$$
\mathcal{H}=\mathcal{H}_{k}+\mathcal{H}_{d}+\mathcal{H}_{m}
$$

At $T=0$,

$$
\begin{aligned}
\mathcal{H}_{k} & =\frac{1}{2 m}\left(\tau_{n}+\tau_{p}\right)=\frac{1}{2 m} \frac{1}{5 \pi^{2} \hbar^{3}}\left(p_{F n}^{5}+p_{F p}^{5}\right) \\
\mathcal{H}_{d} & =\frac{A_{1}}{2 n_{0}} n^{2}+\frac{A_{2}}{2 n_{0}} n^{2}(1-2 x)^{2}+\frac{B}{\sigma+1} \frac{n^{\sigma+1}}{n_{0}^{\sigma}}\left[1-y(1-2 x)^{2}\right] \\
\mathcal{H}_{m} & =\frac{C_{l}}{n_{0}}\left(I_{n n}+I_{p p}\right)+\frac{2 C_{u}}{n_{0}} I_{n p}
\end{aligned}
$$

485

with

$$
\begin{aligned}
x & =n_{p} / n, \quad p_{F i}=\left(3 \pi^{2} n_{i} \hbar^{3}\right)^{1 / 3} \\
I_{i j} & =\frac{8 \pi^{2} \Lambda^{2}}{(2 \pi \hbar)^{6}}\left\{p_{F i} p_{F j}\left(p_{F i}^{2}+p_{F j}^{2}\right)-\frac{p_{F i} p_{F j} \Lambda^{2}}{3}\right. \\
& +\frac{4 \Lambda}{3}\left(p_{F i}^{3}-p_{F j}^{3}\right) \arctan \left(\frac{p_{F i}-p_{F j}}{\Lambda}\right) \\
& -\frac{4 \Lambda}{3}\left(p_{F i}^{3}+p_{F j}^{3}\right) \arctan \left(\frac{p_{F i}+p_{F j}}{\Lambda}\right) \\
& +\left[\frac{\Lambda^{4}}{12}+\frac{\left(p_{F i}^{2}+p_{F j}^{2}\right) \Lambda^{2}}{2}-\frac{\left(p_{F i}^{2}-p_{F j}^{2}\right)^{2}}{4}\right] \\
& \left.\times \ln \left[\frac{\left(p_{F i}+p_{F j}\right)^{2}+\Lambda^{2}}{\left(p_{F i}-p_{F j}\right)^{2}+\Lambda^{2}}\right]\right\} .
\end{aligned}
$$

In this work we use the coefficients $A_{1}=-69.48 \mathrm{MeV}, A_{2}=-29.22 \mathrm{MeV}$, $B=100.1 \mathrm{MeV}, \sigma=1.362, y=-0.0328, C_{l}=-23.06 \mathrm{MeV}, C_{u}=-105.9$ $\mathrm{MeV}$, and $\Lambda=420.9 \mathrm{MeV}[13$.

When a calculation for multiple-species is undertaken, highly asymmetric configurations should be avoided as the various particle types involved will be in different regimes of degeneracy thus resulting in a slower convergence relative to the single-species case. Furthermore, one should also refrain from using multiple species results with $x=0$ or 1 as numerical complications arise; namely, division by 0 occurs in the level density parameters $a_{i}$.

\section{Appendix C. HAMILTONIAN DENSITY FOR SKYRME MODELS}

Taking symmetric nuclear matter (SNM) for illustration, the Hamiltonian density $\mathcal{H}(n, \tau)$ for Skyrme models with zero-range interactions reads as

$$
\mathcal{H}(n, \tau)=\frac{\hbar^{2}}{2 m} \tau+\frac{1}{16}\left[\left(3 t_{1}+5 t_{2}\right)+4 t_{2} x_{2}\right] n \tau+\frac{3}{8} t_{0} n^{2}+\frac{1}{16} t_{3} n^{\epsilon+2},
$$


where $t_{1}$ through $t_{3}$, and $x_{2}$ are dimensionfull strength constants. The first term above is purely kinetic in origin. The second term arises from the exchange soo part of the $p$-wave zero-range interaction [26]. The term $\propto n^{2}$ represents the contribution from two-body $s$-wave zero-range interactions, whereas the last term $\propto n^{2+\epsilon}$ with $\epsilon>0$ accounts for higher than two-body interactions. The functional derivatives of $\mathcal{H}(n, \tau)$ with respect to $n$ and $\tau$ yield the single-particle spectrum [25]:

$$
\begin{aligned}
\varepsilon_{p} & =p^{2} \frac{\partial \mathcal{H}}{\partial \tau}+\frac{\partial \mathcal{H}}{\partial n} \\
& =\frac{p^{2}}{2 m}[1+\beta n]+\frac{3}{4} t_{0} n+\frac{\epsilon+2}{16} t_{3} n^{\epsilon+1}+\frac{1}{16}\left[\left(3 t_{1}+5 t_{2}\right)+4 t_{2} x_{2}\right] \tau \\
& =\frac{p^{2}}{2 m}+R(n, p)+\mathcal{U}(n)=\frac{p^{2}}{2 m^{*}}+\mathcal{U}(n),
\end{aligned}
$$

$n$ are shown separately (having utilized the relations $n=\frac{2 k_{F}^{3}}{3 \pi^{2}}$ and $\tau=\frac{3}{5} k_{F}^{2} n$ in SNM). Equation (C.2 also serves to identify the nucleon's Landau effective mass through

$$
\begin{aligned}
\frac{m^{*}}{m} & =\left[1+\frac{m}{p} \frac{d R(p)}{d p}\right]_{p_{F}}^{-1} \\
& =[1+\beta n]^{-1} \quad \text { with } \quad \beta=\frac{1}{16} \frac{2 m}{\hbar^{2}}\left[\left(3 t_{1}+5 t_{2}\right)+4 t_{2} x_{2}\right] .
\end{aligned}
$$

The structure of $R(n, p)$ in isospin asymmetric matter is similar, albeit with 510 more terms of similar ilk (see, e.g., Ref. [13] for the full expressions in the $\mathrm{SkO}^{\prime}$ and other Skyrme models). The origin of $R(n, p) \propto p^{2}$ in zero-range (in coordinate space) Skyrme models is the exchange part of the $p$-wave interactions which can be subsumed in a density-dependent effective mass $m^{*}$ as the kinetic energy piece is also $\propto p^{2}$. However, derivatives of the Landau effective mass

\section{Appendix D. MEAN-FIELD THEORETICAL MODEL (MFT)}

The MFT model used here involves the exchange of $\sigma, \omega$ and $\rho$ mesons (scalar, vector and iso-vector, respectively) 22. Its Lagrangian density is

$$
\begin{aligned}
\mathcal{L} & =\bar{\Psi}\left[i \gamma_{\mu} \partial^{\mu}-\gamma_{0} g_{\omega} \omega_{0}-\gamma_{0} \frac{g_{\rho}}{2} \rho_{0} \tau_{3}-\left(M-g_{\sigma} \sigma_{0}\right)\right] \Psi \\
& -\frac{1}{2}\left[m_{\sigma}^{2} \sigma_{0}^{2}+\frac{\kappa}{3}\left(g_{\sigma} \sigma_{0}\right)^{3}+\frac{\lambda}{12}\left(g_{\sigma} \sigma_{0}\right)^{4}\right]+\frac{1}{2} m_{\omega}^{2} \omega_{0}^{2}+\frac{1}{2} m_{\rho}^{2} \rho_{0}^{2}
\end{aligned}
$$

which yields the following meson equations of motion:

$$
g_{\sigma}\langle\bar{\Psi} \Psi\rangle=g_{\sigma} n_{S}=m_{\sigma}^{2} \sigma_{0}+\frac{\kappa}{2} g_{\sigma}^{3} \sigma_{0}^{2}+\frac{\lambda}{6} g_{\sigma}^{4} \sigma_{0}^{3}
$$




$$
\begin{aligned}
\omega_{0} & =\frac{g_{\omega}}{m_{\omega}^{2}}\left\langle\Psi^{\dagger} \Psi\right\rangle=\frac{g_{\omega}}{m_{\omega}^{2}} n \\
\rho_{0} & =\frac{g_{\rho}}{2 m_{\rho}^{2}}\left\langle\Psi^{\dagger} \tau_{3} \Psi\right\rangle=\frac{g_{\rho}}{2 m_{\rho}^{2}}\left(n_{n}-n_{p}\right)
\end{aligned}
$$

${ }_{520}$ in the mean-field approximation with classical expectation values denoted by the subscript " 0 ". The equation of motion for the nucleon $(\Psi)$ field is

$$
\left[i \gamma_{\mu} \partial^{\mu}-\gamma_{0}\left(g_{\omega} \omega_{0}+\frac{g_{\rho}}{2} \rho_{0} \tau_{3}\right)-M^{*}\right] \Psi=0
$$

where $M^{*}=M-g_{\sigma} \sigma_{0}$, and yields the nucleon single-particle energy spectrum

$$
\epsilon_{i \pm}= \pm E_{i}^{*}+\frac{g_{\omega}^{2}}{m_{\omega}^{2}} n+\frac{g_{\rho}^{2}}{4 m_{\rho}^{2}}\left(n_{i}-n_{j}\right), \quad E_{i}^{*}=\left(p_{i}^{2}+M^{* 2}\right)^{1 / 2}
$$

The subscripts $i, j$ refer to the nucleon species, the positive sign to the particles and the negative sign to the antiparticles. The thermodynamics of the system are obtained from its energy-momentum tensor

$$
\begin{aligned}
T_{\mu \nu} & =\frac{\partial \mathcal{L}}{\partial\left(\partial_{\mu} \phi\right)} \partial_{\nu} \phi-g_{\mu \nu} \mathcal{L} \\
& =i \bar{\Psi} \gamma_{\mu} \partial_{\nu} \Psi+\frac{g_{\mu \nu}}{2}\left[m_{\sigma}^{2} \sigma_{0}^{2}+\frac{\kappa}{3}\left(g_{\sigma} \sigma_{0}\right)^{3}+\frac{\lambda}{12}\left(g_{\sigma} \sigma_{0}\right)^{4}-m_{\omega}^{2} \omega_{0}^{2}-m_{\rho}^{2} \rho_{0}^{2}\right]
\end{aligned}
$$

For an isotropic system in its rest-frame, the energy density and the pressure are given by the diagonal elements of $T_{\mu \nu}$ as

$$
\begin{aligned}
\varepsilon & =\left\langle T_{00}\right\rangle=2 \sum_{i} \int f_{p_{i}}\left(p_{i}^{2}+M^{* 2}\right)^{1 / 2} \frac{d^{3} p_{i}}{(2 \pi \hbar)^{3}}+\frac{g_{\omega}^{2}}{2 m_{\omega}^{2}} n^{2} \\
& +\frac{g_{\rho}^{2}}{8 m_{\rho}^{2}}\left(n_{p}-n_{n}\right)^{2}+\frac{1}{2}\left[m_{\sigma}^{2} \sigma_{0}^{2}+\frac{\kappa}{3}\left(g_{\sigma} \sigma_{0}\right)^{3}+\frac{\lambda}{12}\left(g_{\sigma} \sigma_{0}\right)^{4}\right] \\
P & =\frac{1}{3}\left\langle T_{i i}\right\rangle=\frac{1}{3} \times 2 \sum_{i} \int f_{p_{i}} \frac{p_{i}^{2}}{\left(p_{i}^{2}+M^{* 2}\right)^{1 / 2}} \frac{d^{3} p_{i}}{(2 \pi \hbar)^{3}}+\frac{g_{\omega}^{2}}{2 m_{\omega}^{2}} n^{2} \\
& +\frac{g_{\rho}^{2}}{8 m_{\rho}^{2}}\left(n_{p}-n_{n}\right)^{2}-\frac{1}{2}\left[m_{\sigma}^{2} \sigma_{0}^{2}+\frac{\kappa}{3}\left(g_{\sigma} \sigma_{0}\right)^{3}+\frac{\lambda}{12}\left(g_{\sigma} \sigma_{0}\right)^{4}\right]
\end{aligned}
$$

The minimization of the grand potential $\Omega=-P V$ with respect to $\sigma_{0}$ (equivalent to $\partial \varepsilon / \partial \sigma_{0}=0$ at $T=0$ and to $\partial P / \partial \sigma_{0}=0$ at finite temperature) leads to a self-consistent equation for the Dirac effective mass

$$
M^{*}=M-\frac{g_{\sigma}^{2}}{m_{\sigma}^{2}}\left[n_{s}-\frac{\kappa}{2}\left(M-M^{*}\right)^{2}-\frac{\lambda}{6}\left(M-M^{*}\right)^{3}\right] .
$$

In the present work we use the masses $M=939.0 \mathrm{MeV}, m_{\sigma}=511.2 \mathrm{MeV}$, $m_{\omega}=783.0 \mathrm{MeV}, m_{\rho}=770.0 \mathrm{MeV}$ and the couplings $g_{\sigma}=9.061, g_{\omega}=10.55$, 
$g_{\rho}=7.475, \kappa=9.194 \mathrm{MeV}, \lambda=-3.280 \times 10^{-2}$. These correspond to a cold symmetric nuclear matter equilibrium density $n_{0}=0.155 \mathrm{fm}^{-3}$ at which the energy per particle $E / A=-16 \mathrm{MeV}$, the compression modulus $K_{0}=225 \mathrm{MeV}$, and the symmetry energy $S_{v}=30 \mathrm{MeV}$.

\section{References}

[1] G. Baym, C. Pethick, Landau Fermi-Liquid Theory, Wiley Interscience, New York, 1991.

[2] A. Burrows, J. M. Lattimer, The birth of neutron stars, Astrophys. J.307 (1986) 178-196. doi:10.1086/164405.

[3] A. Burrows, Supernova neutrinos, Astrophys. J.334 (1988) 891-908. doi: $10.1086 / 166885$.

[4] Y. Sekiguchi, K. Kiuchi, K. Kyutoku, M. Shibata, Gravitational waves and neutrino emission from the merger of binary neutron stars, Phys. Rev. Lett. 107 (2011) 051102. doi:10.1103/PhysRevLett.107.051102. URL http://link.aps.org/doi/10.1103/PhysRevLett.107.051102

[5] L. Landau, E. M. Lifshitz, Statistical Physics, Volume 5, Part 1, 3rd Edition, Pergamon, New York, 1980.

[6] E. M. Lifshitz, L. P. Pitaevskii, Statistical Physics Part 2, Butterworth Heinemann, Oxford, 1980.

[7] G. Baym, S. A. Chin, Landau theory of relativistic fermi liquids, Nuclear Physics A 262 (3) $\quad$ (1976) $527 \quad-\quad 538$. http://dx.doi.org/http://dx.doi.org/10.1016/0375-9474(76)90513-3

555 doi:http://dx.doi.org/10.1016/0375-9474(76)90513-3.

1] URL http://www.sciencedirect.com/science/article/pii/ 0375947476905133

[8] C. J. Pethick, G. M. Carneiro, Specific heat of a normal fermi liquid. i. landau-theory approach, Phys. Rev. A 7 (1973) 304-318. doi:10.1103/ PhysRevA.7.304.

URL http://link.aps.org/doi/10.1103/PhysRevA.7.304

[9] J. Blaizot, B. Friman, On the nucleon effective mass in nu-

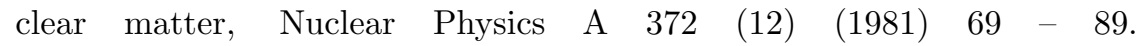
http://dx.doi.org/http://dx.doi.org/10.1016/0375-9474(81)90087-7

565 doi:http://dx.doi.org/10.1016/0375-9474(81)90087-7.

n URL http://wwW.sciencedirect.com/science/article/pii/ 0375947481900877

[10] D. Coffey, K. S. Bedell, Nonanalytic contributions to the self-energy and the thermodynamics of two-dimensional fermi liquids, Phys. Rev. Lett. 71 (1993) 1043-1046. doi:10.1103/PhysRevLett.71.1043 URL http://link.aps.org/doi/10.1103/PhysRevLett.71.1043 
[11] C. Mahaux, P. Bortignon, R. Broglia, C. Dasso, Dynamics of the shell model, Physics Reports 120 (1) (1985) 1 - 274. http://dx.doi.org/http://dx.doi.org/10.1016/0370-1573(85)90100-0 doi:http://dx.doi.org/10.1016/0370-1573(85)90100-0.

URL 0370157385901000

[12] S. Fantoni, B. Friman, V. Pandharipande, Correlated basis theory of nucleon optical potential in nuclear matter, Nuclear Physics A 399 (1) (1983) 51 - 65. http://dx.doi.org/http://dx.doi.org/10.1016/0375-9474(83)905936 doi:http://dx.doi.org/10.1016/0375-9474(83)90593-6. URL http://www.sciencedirect.com/science/article/pii/ 0375947483905936

[13] C. Constantinou, B. Muccioli, M. Prakash, J. M. Lattimer, Thermal properties of hot and dense matter with finite range interactions, Phys. Rev. C 92 (2015) 025801. doi:10.1103/PhysRevC.92.025801. URL http://link.aps.org/doi/10.1103/PhysRevC.92.025801

[14] S. Hama, B. C. Clark, E. D. Cooper, H. S. Sherif, R. L. Mercer, Global dirac optical potentials for elastic proton scattering from heavy nuclei, Phys. Rev. C 41 (1990) 2737-2755. doi:10.1103/PhysRevC.41.2737. URL http://link.aps.org/doi/10.1103/PhysRevC.41.2737

[15] P. Danielewicz, R. Lacey, W. G. Lynch, Determination of the equation of state of dense matter, Science 298 (5598) (2002) 1592-1596. http://arxiv.org/abs/http://www.sciencemag.org/content/298/5598/1592.full.pdf arXiv:http://www.sciencemag.org/content/298/5598/1592.full. pdf, doi:10.1126/science.1078070. URL http://www. sciencemag.org/content/298/5598/1592. abstract

[16] P. Demorest, T. Pennucci, S. Ransom, M. Roberts, J. Hessels, A two-solarmass neutron star measured using shapiro delay, Nature 467 (7319) (2010) 1081-1083. doi:10.1038/nature09466.

[17] J. Antoniadis, P. C. C. Freire, N. Wex, T. M. Tauris, R. S. Lynch, M. H. van Kerkwijk, M. Kramer, C. Bassa, V. S. Dhillon, T. Driebe, J. W. T. Hessels, V. M. Kaspi, V. I. Kondratiev, N. Langer, T. R. Marsh, M. A. McLaughlin, T. T. Pennucci, S. M. Ransom, I. H. Stairs, J. van Leeuwen, J. P. W. Verbiest, D. G. Whelan, A massive pulsar in a compact relativistic binary, Science 340 (6131). doi:10.1126/science.1233232.

a URL http://www.sciencemag.org/content/340/6131/1233232. abstract

[18] G. M. Welke, M. Prakash, T. T. S. Kuo, S. Das Gupta, C. Gale, Azimuthal distributions in heavy ion collisions and the nuclear equation of state, Phys. Rev. C 38 (1988) 2101-2107. doi:10.1103/PhysRevC.38.2101. URL http://link .aps.org/doi/10.1103/PhysRevC.38.2101 
[19] C. B. Das, S. Das Gupta, C. Gale, B.-A. Li, Momentum dependence of symmetry potential in asymmetric nuclear matter for transport model calculations, Phys. Rev. C 67 (2003) 034611. doi:10.1103/PhysRevC.67.034611. URL http://link.aps.org/doi/10.1103/PhysRevC.67.034611

[20] P.-G. Reinhard, D. J. Dean, W. Nazarewicz, J. Dobaczewski, J. A. Maruhn,

M. R. Strayer, Shape coexistence and the effective nucleon-nucleon interaction, Phys. Rev. C 60 (1999) 014316. doi:10.1103/PhysRevC.60.014316 URL http://link.aps.org/doi/10.1103/PhysRevC.60.014316

[21] M. Prakash, T. T. S. Kuo, S. Das Gupta, Momentum dependence, boltzmann-uehling-uhlenbeck calculations, and transverse momenta, Phys. Rev. C 37 (1988) 2253-2256. doi:10.1103/PhysRevC.37.2253. URL http://link.aps.org/doi/10.1103/PhysRevC.37.2253

[22] H. Müller, B. D. Serot, Relativistic mean-field theory and the high-density nuclear equation of state, Nuclear Physics A 606 (1996) 508-537. arXiv: nucl-th/9603037, http://dx.doi.org/10.1016/0375-9474(96)00187-X doi : 10.1016/0375-9474(96)00187-X.

[23] J. Kapusta, Finite-Temperature Field Theory, Cambridge Monographs on Mathematical Physics, Cambridge University Press, 1993. URL https://books .google.com/books?id=nT2kbcDtMl8C

[24] S. Johns, P. J. Ellis, J. Lattimer, Numerical approximation to the thermodynamic integrals, The Astrophysical Journal 473 (2) (1996) 1020. doi:10.1086/178212

[25] C. Constantinou, B. Muccioli, M. Prakash, J. M. Lattimer, Thermal properties of supernova matter: The bulk homogeneous phase, Phys. Rev. C 89 (2014) 065802. doi:10.1103/PhysRevC.89.065802. URL http://link.aps .org/doi/10.1103/PhysRevC.89.065802

[26] D. Vautherin, D. M. Brink, Hartree-fock calculations with skyrme's in640 teraction. i. spherical nuclei, Phys. Rev. C 5 (1972) 626-647. doi: 10.1103/PhysRevC.5.626.

URL http://link .aps.org/doi/10.1103/PhysRevC.5.626 


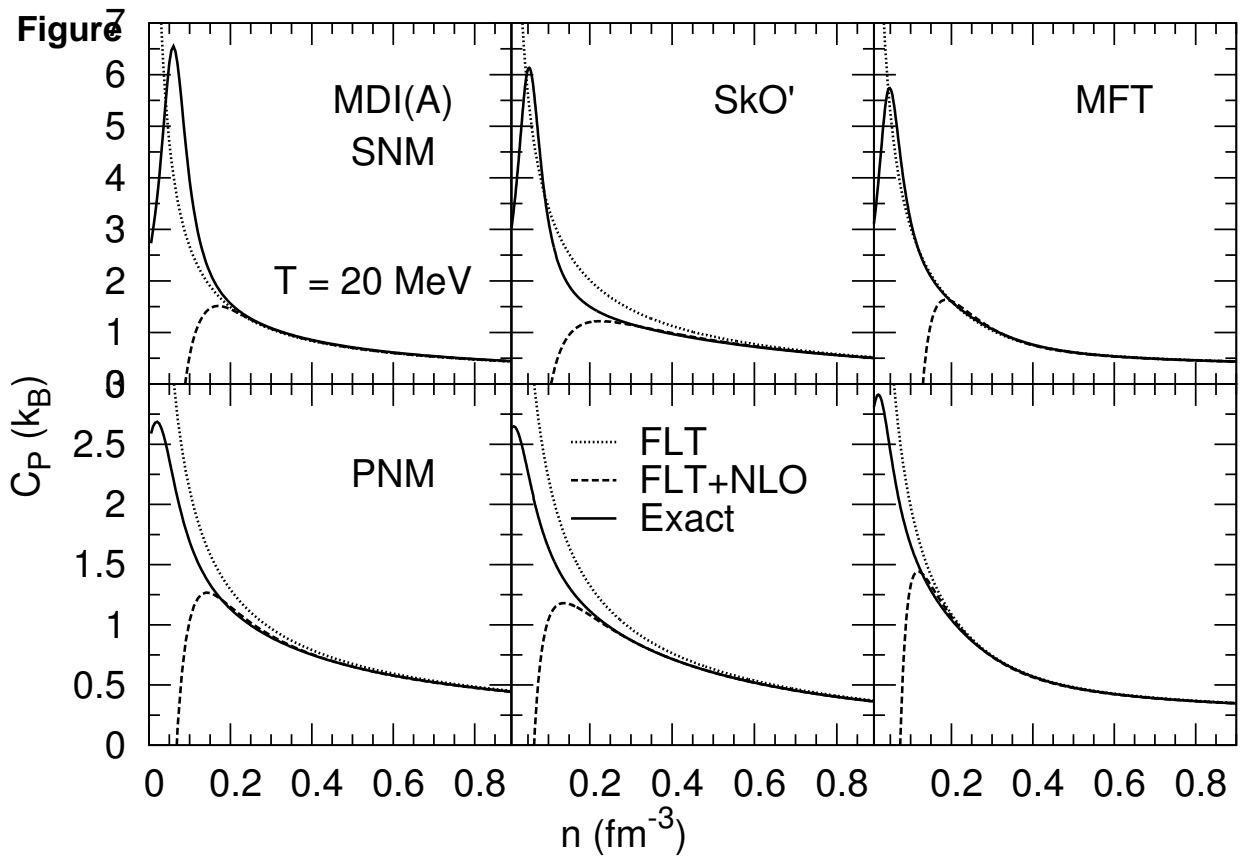




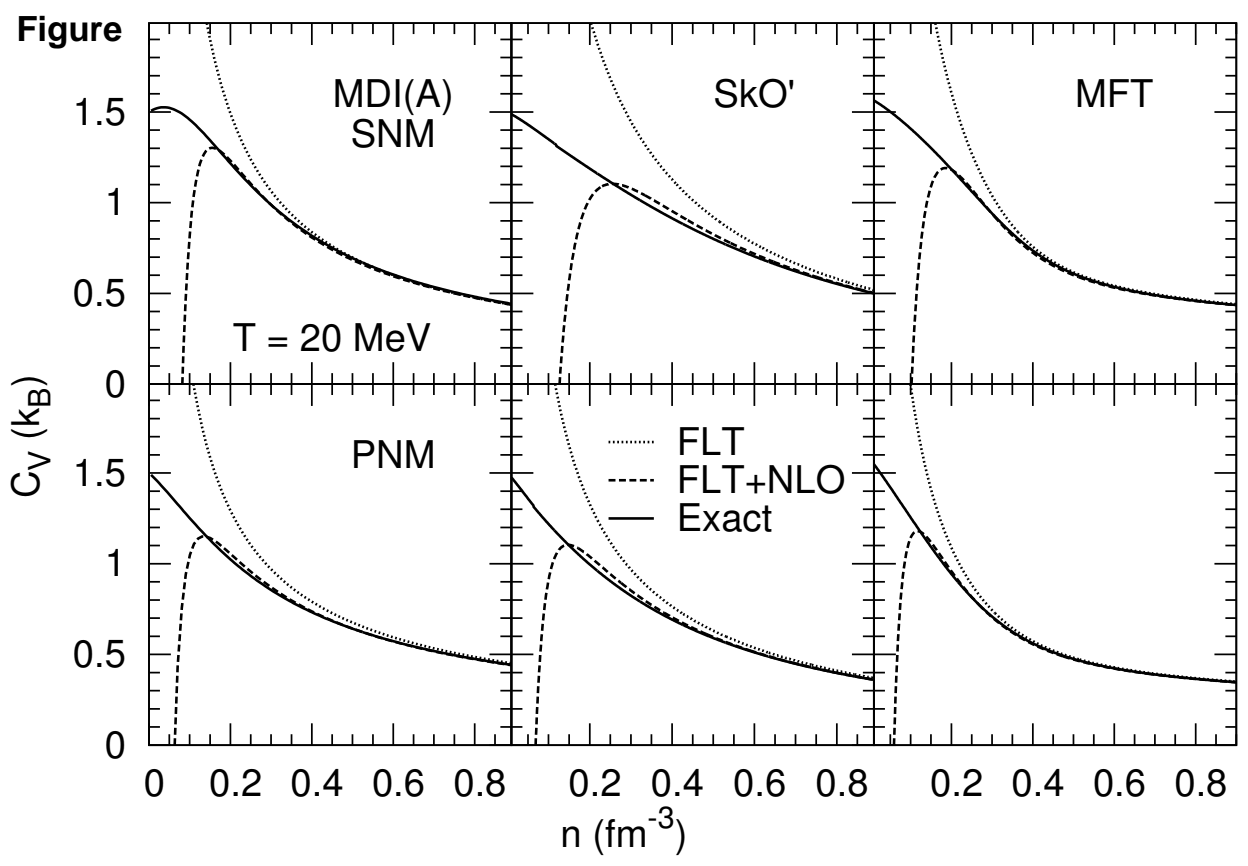




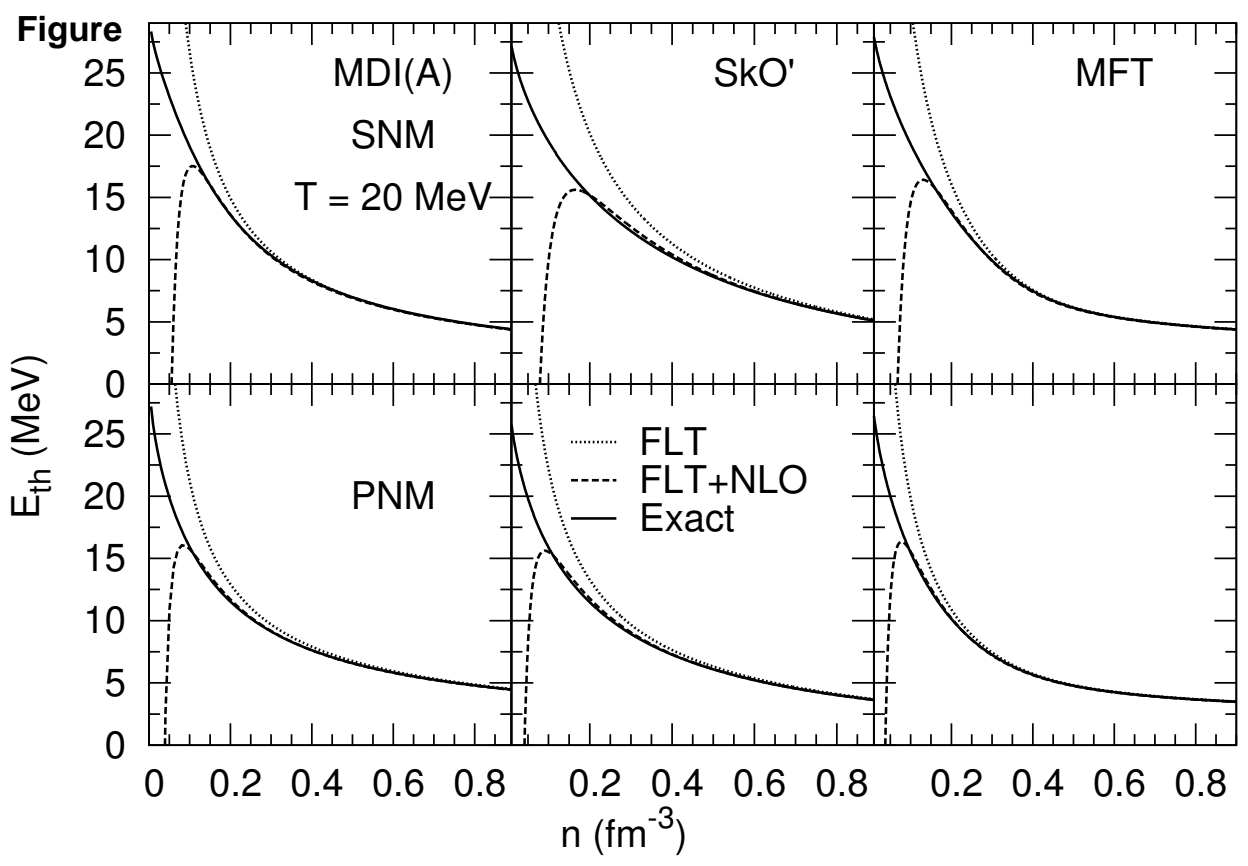




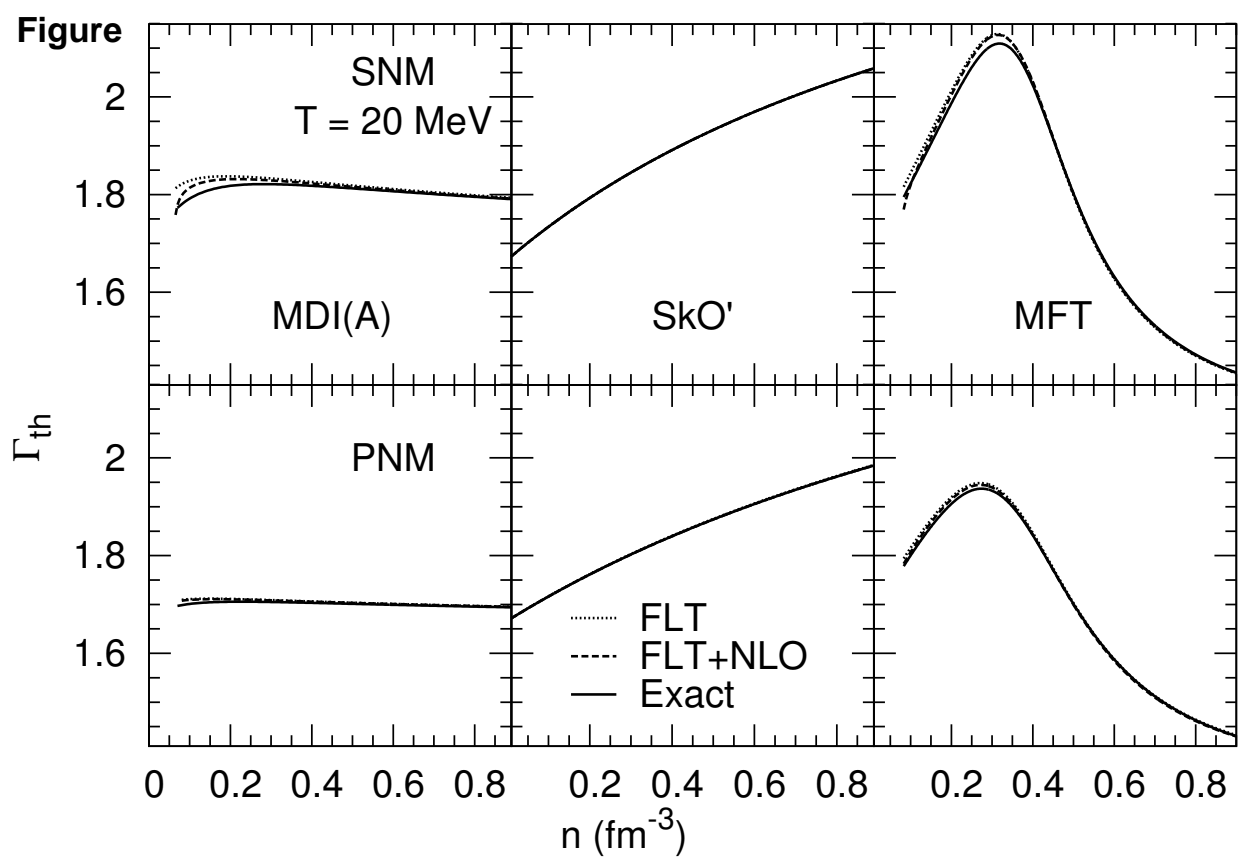




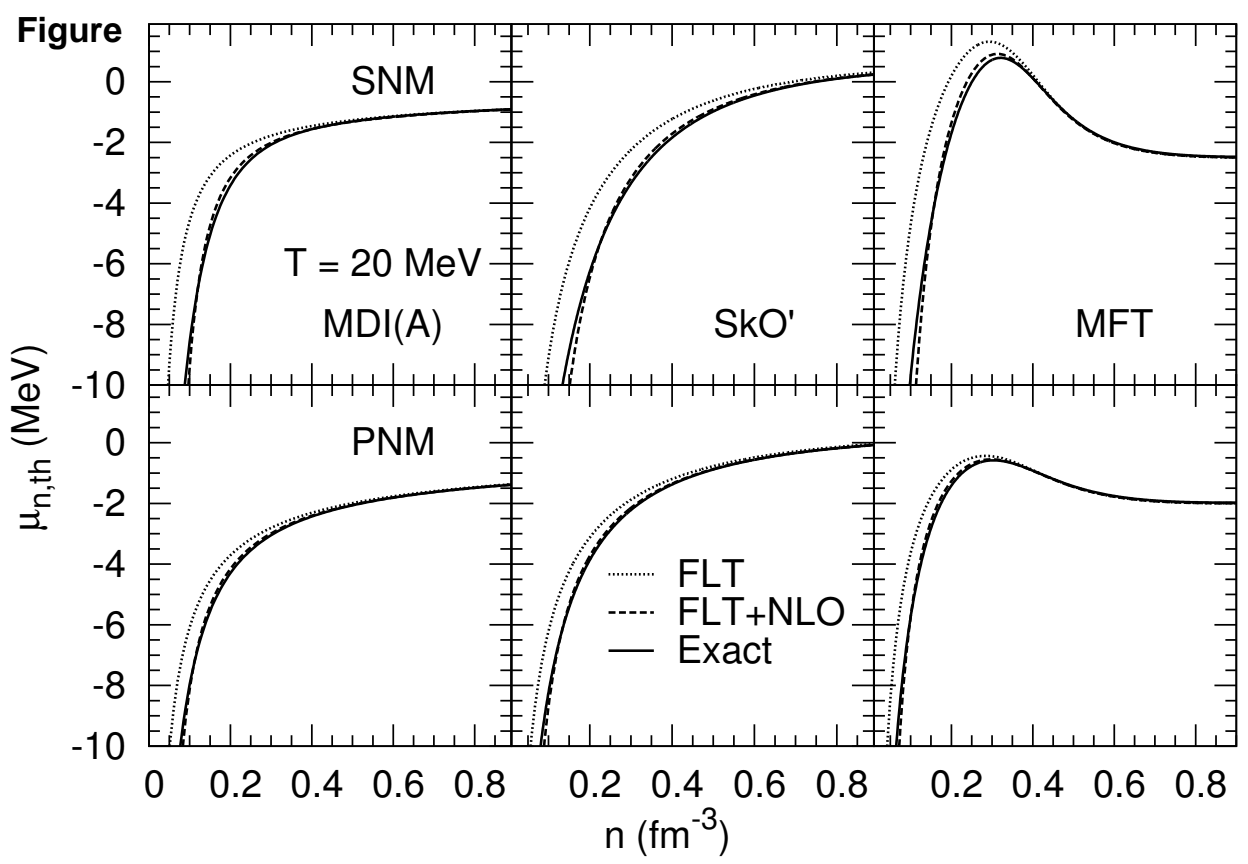




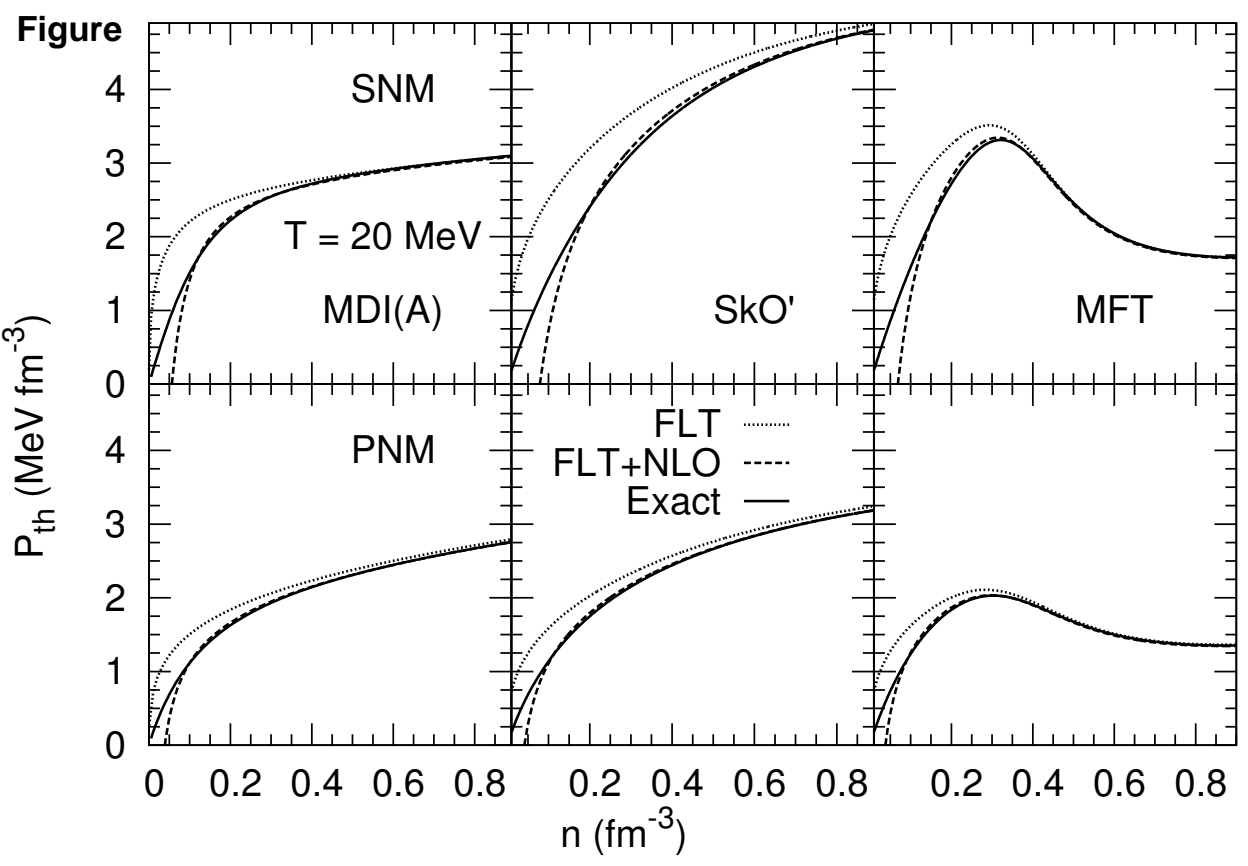




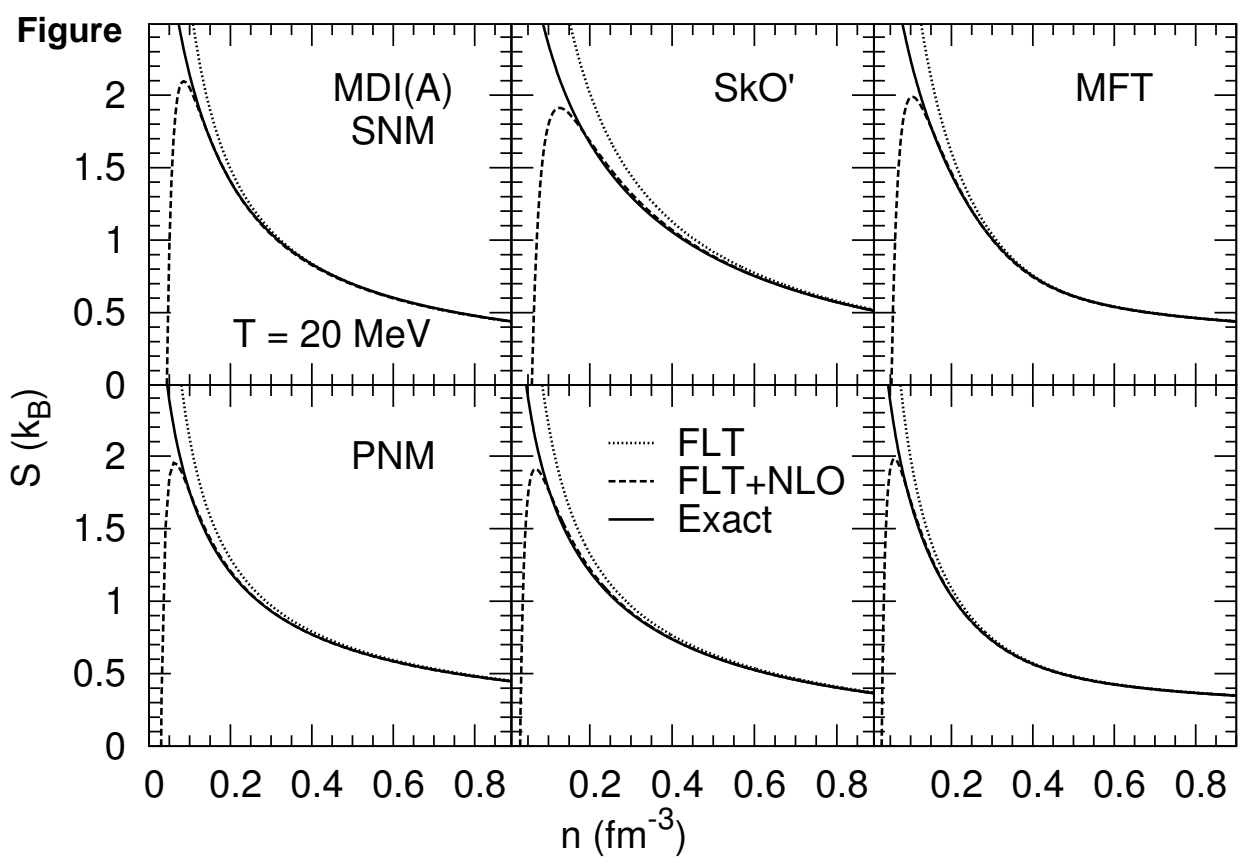

